Portland State University

PDXScholar

3-1997

\title{
Tool Condition Monitoring: A Computational Approach
}

Anthony Clinch

Portland State University

Follow this and additional works at: https://pdxscholar.library.pdx.edu/open_access_etds

Part of the Mechanical Engineering Commons

Let us know how access to this document benefits you.

\section{Recommended Citation}

Clinch, Anthony, "Tool Condition Monitoring: A Computational Approach" (1997). Dissertations and Theses. Paper 5728.

https://doi.org/10.15760/etd.7599

This Thesis is brought to you for free and open access. It has been accepted for inclusion in Dissertations and Theses by an authorized administrator of PDXScholar. Please contact us if we can make this document more accessible: pdxscholar@pdx.edu. 
The abstract and thesis of Anthony Clinch for the Master of Science in Mechanical Engineering were presented November 15,1996 , and accepted by the thesis committee and the department.

COMMITTEE APPROVALS:

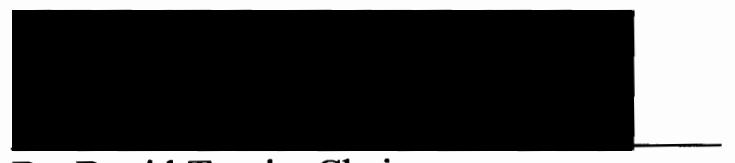

Dr. David Turcic, Chair

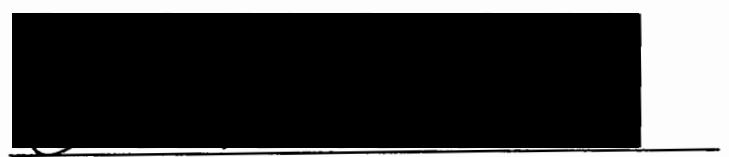

Dr. Gerald Recktenwald

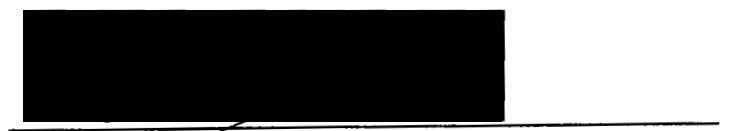

Dr. Chien Wern
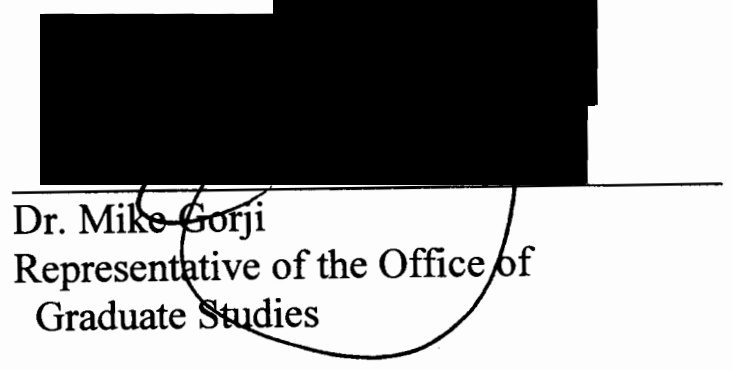

DEPARTMENT APPROVAL:

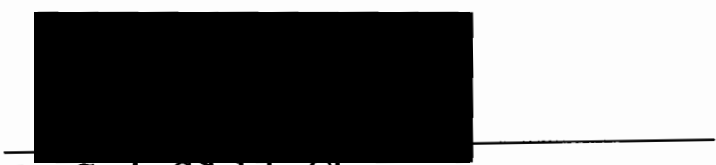

Dr. Graig Spolek, Chair

Department of Mechanical Engineering

ACCEPTED FOR PORTLAND STATE UNIVERSITY BY THE LIBRARY

by on II March 1997 


\section{Abstract}

An abstract of the thesis of Anthony Clinch for the Master of Science in Mechanical Engineering, presented November $15^{\text {th }} 1996$.

Title: Tool Condition Monitoring. A Computational Approach.

Tool Condition Monitoring offers a new approach to reducing the costs associated with catastrophic tool failure. The manufacturing process is becoming more computationally oriented all the time. Computational methods of determining the condition of cutting tools will lend themselves to easy integration with current and forthcoming manufacturing techniques. Coupling the results of this computational approach with a cost analysis will provide a means of maximizing the investment in cutting tools, while minimizing the damage caused by tool failure.

Tool Condition Monitoring, as defined here, is a method of computationally determining the condition of a cutting tool. The problem of determining a tool's condition is approached by geometrically modeling the cutting process using a solid modeling software package. From a model of a process, an approximation of the forces that act on a cutting tool during that process are made. The calculated cutting forces are used to generate a stress history of the cutting tool, which can be maintained in a database. Statistical methods can then be applied to the stress history data to determine the probability that a cutting tool will fail at any point in a cutting process. 
The main purpose of this work is to develop an approach to predicting the condition of a cutting tool based on solid geometric models of cutting processes. The result of this work is an algorithm that describes how models are set up and executed to produce a set of intersections called chips. The algorithm continues by processing the chips to compute the cutting forces acting on the nodes of a finite element mesh of a cutting tool. The forces generated from these routines are used by a finite element analysis software package to produce a history of the stresses that occur in a tool. From this information, the condition of the cutting tool can be approximated using statistical methods. 


\title{
TOOL CONDITION MONITORING,
}

\section{A COMPUTATIONAL APPROACH}

\author{
by \\ Anthony Clinch
}

A thesis submitted in partial fulfillment of the requirements for the degree of

\author{
MASTER OF SCIENCE \\ in \\ MECHANICAL ENGINEERING
}

Portland State University

1997 


\section{Table Of Contents}

1. Introduction

1.1 Background 3

1.2 Tool Condition Monitoring 5

1.3 The ACIS 3DToolkit 8

1.4 Overview 10

2. Conversion of Mesh Data 15

2.1 Introduction 15

2.2 Preparing The Data 16

2.3 Elements as Solids 21

2.4 Limitations 27

3. Modeling A Cutting Process 28

3.1 Introduction 28

3.2 Tool Construction 31

3.3 Transformations 34

3.4 Model One 37

3.5 Model Two 39

3.6 Chip Generation. 42 
4.1 Introduction 45

4.2 The Direction of the Cutting Force 48

4.3 The Magnitude of the Cutting Force 52

4.4 Algorithmic Details of Force Calculations 53

4.5 Storage of information as attributes 55

5. Results 58

5.1 Introduction 58

5.2 Model One 61

5.3 Change in Direction of Cutting Force 69

5.4 Model Two 75

5.5 Conclusion 81 


\section{Introduction}

\subsection{Background}

Work related to the understanding and improvement of the cutting process was under way prior to the Industrial Revolution. During the Industrial Revolution an enormous amount of work was done to improve and develop machine tools [1]. Most of this work occurred in a trial and error manner. By the later part of the $18^{\text {th }}$ century the machine tool industry had greatly matured. Efforts were made to optimize the cutting process, determining the minimum number of passes needed to produce a given part [2]. Major innovations, such as the use of high speed steels, and variable speed machines, had greatly increased the efficiency of machine shops by the end of the Nineteenth Century.

In the latter half of the Twentieth Century automation of machine tools has further increased the efficiency of machine shops and made the fabrication of more complex parts much easier. With all of the advancements of the machining process to date, catastrophic failure of cutting tools still occurs. Tool failure is possibly the largest source of inefficiency remaining in the cutting process. Although automation of the machining process makes unattended operation possible, tool failure can make unattended operation a risky procedure. 
Historically, understanding the problem of tool failure was approached by observing the types of failures and the conditions under which those failures occurred. From these observations, classifications were defined so that tool failures could be understood. As experimental techniques have become more sophisticated, so have the analysis of cutting tool failure. A large amount of effort has gone into the analysis of cutting tools and the conditions under which they fail [3]. This type of work has continued to improve the understanding of the cutting process, as well as increase the efficiency of that process.

In addition to the analysis of tool failure, efforts have been made to predict tool failure by observing variables of a cutting process, such as the frequency of acoustic emissions, so that changes in these variables, which may indicate tool failure, can be used to halt the process [4]. In this manner, automated cutting processes may be able to be automatically terminated prior to tool failure occurring. Preventing tool failure is most cost effective near the end of a machining process, when relatively large amounts of time have gone into the manufacturing of a part. Tool failure and the damage that may result to the work piece, can be far more expensive than the cost of a replacement tool. In addition to the damage done to the work piece, the safety of shop personnel and added down time of the machine are costly side effects to be considered in the discussion of tool failure. 
With the advent of solid modeling techniques it is now possible to easily represent and manipulate models of solid bodies in the space of computer memory. Solid modeling techniques have been used for the purpose of modeling a machining processes to verify that the NC machining processes is correct [5]. In addition to using the information embodied in solid models that result from modeling a cutting process for verification of solid geometry, it is also possible to use this information for the purpose of determining the condition of a cutting tool. The solid geometry resulting from modeling a cutting process can be used to approximate the stresses that occur in a cutting tool during a given process.

\subsection{Tool Condition Monitoring}

Tool Condition Monitoring offers at least a partial solution to the problem of catastrophic tool failure. Tool condition monitoring, as described here, is the process of computationally determining the condition of a cutting tool. The goal of tool condition monitoring is to correctly determine the condition of a cutting tool based on the computed stress history of that tool. From the stress history of a tool, a statistical approximation of its condition can be made. From the tool's computed condition, the risk of tool failure during a future cutting process can be analyzed so that the investment in the tool can be maximized and the risk of damage due to tool failure, minimized. Within the scope of a computational method of this type, lies the goal of properly modeling a cutting process to produce a stress history. This work develops an 
algorithm for modeling cutting processes and approximating the cutting forces using solid geometry. In the development of the algorithm, simplifications were made. The method by which the forces were calculated is very simple. The calculated forces are proportional to the volume of material removed. The simplified force calculation function served to facilitate the coding of the complete algorithm, which is the main purpose of this work, and can be replaced by a more accurate method in future work.

A computational method of predicting tool failure has the potential of maximizing the investment in cutting tools, while minimizing costs associated with tool failure. By keeping track of the amount of stress a cutting tool has undergone during its lifetime, statistical methods can be applied to determine the probability that a tool will fail during a process to be completed in the future. The stresses that will occur in a tool can be closely approximated prior to performing the actual cutting process. From the computed stress history of a tool, including the process yet to be physically completed, and a set of data based on experimentally determined cutting tool failures, it is possible to determine a probability that a tool will fail at any point in the process.

The experimental determination of cutting forces in the laboratory is not easy. Very exacting efforts have to be made in order to accurately measure the magnitude and direction of cutting forces. Typically, these measured forces must be resolved into components that act over areas of the tool face and flank. Another difficulty in 
laboratory measurement of cutting forces is the determination of the area over which the forces act. Beyond the analysis performed by specialized individuals [6], on specific cutting processes, this method of determining tool forces may not be generally possible on the shop floor. Computational methods of determine cutting forces stand to be easily integrated into modern machine tools by the addition of programming to a process which presently incorporates computational equipment and methods.

An implementation of the Tool Condition Monitoring algorithm would proceed as follows. Prior to a machine starting a new cutting process, a program would be executed to determine the stresses that would occur in the cutting tool during that process. The stress history from the process to be performed would then be added to the tool's existing stress history so that the probability that the tool will fail can be determined. This information could then be used in a cost analysis to determine if the tool should be used. A tool that may be to risky to use for process X, may have enough useful life left to perform other less risky processes. By performing this type of analysis, the investment in the tool would be maximized.

The process of determining the condition of a cutting tool can be described by the basic steps shown in Figure 1. 


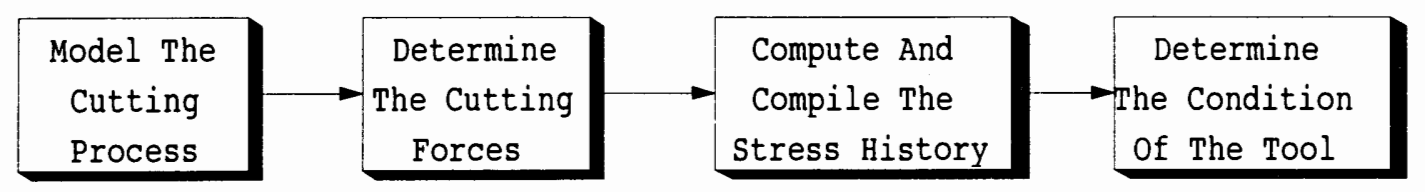

Figure 1 - Flow chart of tool condition monitoring algorithm.

The goal of this thesis was to develop and code an algorithm to facilitate the first two blocks shown in Figure 1. Two simple cutting models were used to develop the algorithm and verify the code. The solid modeling software used to accomplish this task was the ACIS 3DToolkit by Spatial Technology Inc..

\subsection{The ACIS 3DToolkit}

The ACIS 3DToolkit, referred to from this point as the $3 D$ Toolkit, is a an interface to a set of solid modeling routines. The 3DToolkit provides two means by which the solid modeling routines can be accessed, the $\mathrm{C}++$ programming language or the Scheme programming language. The Scheme interface the 3DToolkit was chosen to access the solid modeling routines used here. Scheme is a dialect of the Lisp programming language. Unlike $\mathrm{C}++$, Scheme programs are compiled at run time through a Scheme interpreter. A Scheme interpreter appears to the user as a command line from which calls can be made to functions that are part of the Scheme environment. Scheme is a small, powerful and most importantly, extendible programming language. 
The Scheme interface to the 3DToolkit provides a work place in which algorithms can be easily developed and tested. Scheme is spawned with an initial global environment which can be thought of as a table containing variables and values bound to those variables. In Scheme, the value of a variable can be a procedure as easily as it can be a number or a string [7]. The version of Scheme that the 3DToolkit is based on, has been extended to include a large amount of solid modeling functionality, that can be accessed from the command line of the interpreter. When the 3DToolkit is opened, the initial global environment includes the solid modeling procedures in the global environment table. As algorithms are developed and tested in the interpreter, the environment is expanded to include these new bindings. The $3 D T o o l k i t$ combines the robust solid modeling routines of $A C I S$ with the power of a high level programming language, to create a powerful solid modeling tool.

Version 1.2 of the 3 DToolkit was extended further to include a function for the construction of the elements of a finite element mesh as solid bodies. This extension of the 3DToolkit involved modification of the source code and recompilation to produce a new $3 D$ Toolkit executable. The function used to create the finite elements as solids was named "solid:convert", and is discussed in some detail below. 


\subsection{Overview}

The analysis starts with the definition and construction of a model. A simple cutting tool was constructed in the finite element package ANSYS, where it was meshed. The mesh information was saved to a set of data files. The data consisted of element, node, and position information which described a finite element mesh of the cutting tool. The meshed data was converted to a format favorable to the $3 D$ Toolkit for construction of the meshed elements as solid bodies in the solid modeling environment. The meshed version of the tool was used to determine the forces acting on the nodes of the finite elements. The meshed version of the tool, in the solid modeling environment, is referred to as the tool having multiple lumps. An unmeshed version of the tool was also constructed in the solid modeling environment, and was used to generate the chips that are the result of modeling the cutting process. The unmeshed version of the cutting tool is referred to as the one lump version. A model of a cutting process consists of a piece of stock to be machined, a one lump cutting tool and a set of transformations that define the cutting process or relative path that the tool will follow to perform the process.

The determination of the cutting forces requires several steps. The first step is to run the model of the cutting process and generate a set of chips. The model is run by applying the transformations to the one lump version of the tool along the cutting path and performing Boolean operations to generate the chips, or volumes of intersection of the tool and the stock. A single chip represents a discrete instance in time, for which 
the forces on the tool will be calculated. The chips are generated and saved to a file for use in the determination of the forces acting on the cutting tool. Execution of the routines that calculate the forces involves the intersection of the chips, one at a time, with each of the elements in the meshed version of the tool. From these volumes of intersection of the elements and a chip (referred to as subchips), the magnitudes of the forces acting on the nodes of the elements are calculated. The direction of the cutting force is approximated by the path of the centroid of the chip between two consecutive time-steps. Specifically, a three dimensional direction vector is determined from the centroid of a chip, one time-step prior to the generation of that chip, to the centroid of the chip in the position in space in which it is generated. The determination of the direction vector takes place after the chips have been generated.

The construction of the model can take place in any position and orientation in space. The forces generated by the algorithm however, must be determined in nodal space so that they can be properly applied to the tool in the finite element package. In order to get the chips properly aligned with the multi-lump version of the cutting tool, a set of transformations have to be applied to each of the chips. By transforming the chips onto the meshed version of the tool, all of the positional information is in nodal space. When the model is run, the chips are stored in the positions in which they were generated in model space, which is generally not the same as nodal space. Two sets of transformations are used in order to align the chips with the multi-lump version of the tool. The first of these sets is an inverse set of transformations, of the set used to 
describe the cutting process. These transformations are applied to the chips in order to place them in the orientation of the cutting tool at the start of the cutting process. The orientation of the tool at the start of the cutting process is referred to as the tool's datum position. The direction vector is determined during the final transformation of each of the chips to the datum position, and is unitized. From this datum position a set of transformations are defined to align the chips and the direction vector, with the multi-lump version of the cutting tool, so that the nodal weights can be calculated, and used to scale the direction vector.

The magnitude of the force acting on each of the nodes of the finite element mesh is based on the volume of the subchip and the distance from the node, for which the force is to be determined, to the centroid of the subchip. Each nodal force determined from the subchip is normalized by the volume of the subchip, so that the sum of the magnitudes of the forces acting on the nodes of a given finite element, is proportional to the volume of the subchip for that element. After all of the nodal weights have been calculated for a single chip, the weights for nodes that have adjacent elements are summed. Finally, the direction vector is scaled from the each of the node positions by the respective nodal weights in nodal space. The forcing information for each chips is compiled and written to a file in a format that allows it to be imported into the finite element software package. The result of this process, is a set of node numbers and respective cutting force components in $\mathrm{x}, \mathrm{y}$, and $\mathrm{z}$. A set of nodal forces are determined for each chip. The result of processing all of the chips produces 
a discrete force history based on the solid geometry of a tool, stock and their intersection. The process of generating the cutting forces can be divided into four main tasks. Figure 2 outlines the process of generating the cutting forces, in the form of a flow chart.

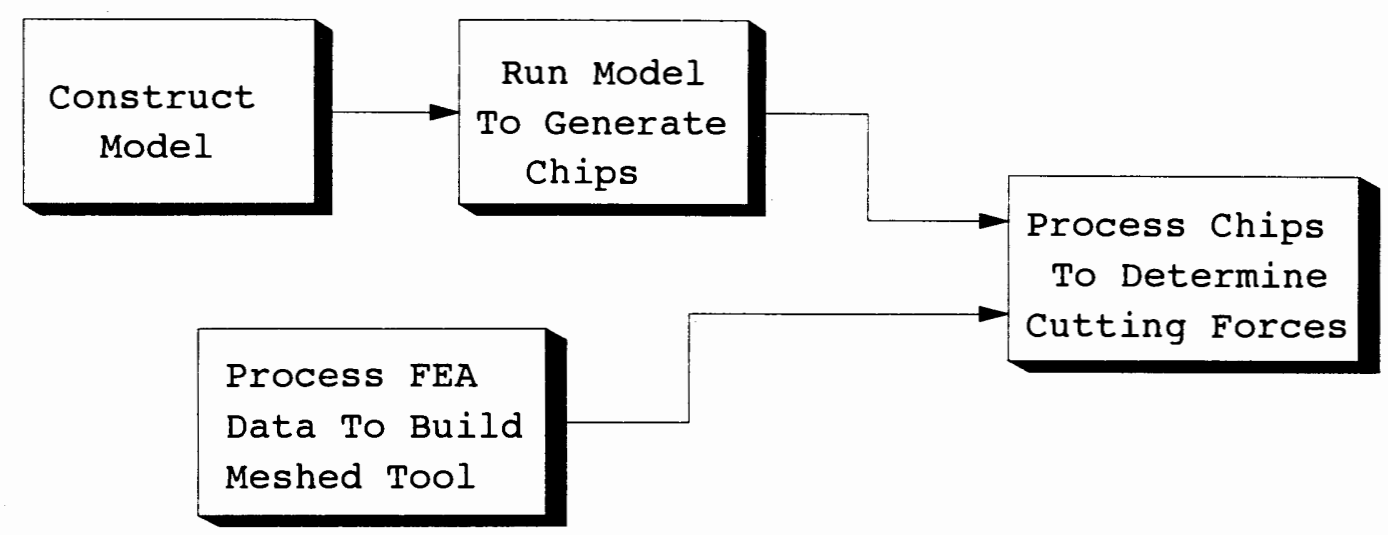

Figure 2 - Flow chart of model construction and force generation.

For the purpose of developing the code, each of these four steps was considered a separate process, for which the programs were developed to run separately. After the chips have been generated by running a model, they are saved to a file and restored during the routines that calculate the forces acting on the nodes of the finite element mesh. The meshed version of the cutting tool is also constructed and saved to a part file, from which it can be restored. Keeping the routines separate, in this manner, allows alternate tool meshes to be introduced, without having to re-run 
the model of the cutting process. The same set of chips can be used to calculate the nodal forces for different tool meshes.

The calculation of the cutting forces was the most computationally intensive part of this process. The ability to group the chips into several part files can be exploited as a means of breaking up a large cutting process into a set of smaller ones. This will provide one means of dealing with the large demand that solids modeling places on system resources. Another approach to dealing with the computational load of calculating cutting forces, would be to focus the calculations on areas of the cutting process that are changing, such as the engagement and disengagement of a tool with a work piece. In this manner a small set of calculations could be done in sections of the cutting process that are constant, and could be applied to that entire section. 


\section{Conversion of Mesh Data}

\subsection{Introduction}

Calculation of the stress field in the cutting tool, on a large scale, is made possible via the use of finite element analysis (FEA) software. Before the forces acting on the nodes of the meshed model of the cutting tool can be calculated, the mesh must be imported into the solid modeling software by creating solid lumps that represent the finite elements. The manipulations which were applied to the finite element mesh data, and the construction of the finite elements as solids is described below.

The format in which the mesh data was generated dictated a couple of issues which had to be addressed when preparing the data for the solids modeler. The first of those issues occurred because only the area of the mesh around the tip of the tool was to be constructed in the solids modeler. Minimizing the size of the mesh allows for a reduction in the computational load required to calculate the forces. The problem caused by using a subset of elements is that the element data file is minimized, but the node data file contains all of the information of the tool mesh. The second issue in converting the mesh data is caused by the fact that the finite element software and the solids modeling software required the node data in a different orders. Because of this the node data had to be transformed before use by the solids modeler and then 
transformed back to the original order before use by the finite element package. The general algorithm for converting tool mesh data to solid elements is shown in Figure 3.

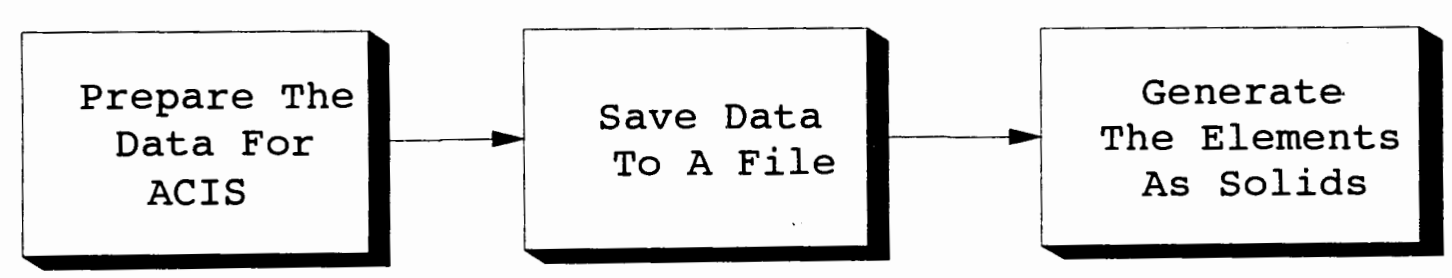

Figure 3 - Flow chart of tool mesh conversion to solid element.

\subsection{Preparing The Data}

Having defined the specifications of the cutting tool, a model of the tool was constructed and meshed in a FEA software package. In this case, the FEA package used to generate the mesh of the tool was ANSYS. The tool mesh data was exported from ANSYS in the form of data files. The data generated by ANSYS had to be manipulated before it could be used to generate the solid elements in the solid modeling software package. The original form of the mesh data was contained in two files. One of the two files contained the element information, which consisted of a list of the element numbers and the nodes numbers for each of the elements. The second file contained the nodal information consisting of the node numbers and the positions of the nodes in nodal space. The first step in processing the data was to combine the two files into a single file which completely described the finite element mesh while 
completely preserving the original mesh data. The single data file was used to build the elements as solids.

The information contained in the two data files was combined into one file containing each of the element numbers and respective positions of the nodes. The steps taken to convert the mesh data, to a format useful for generating the elements as solids, are shown in Figure 4.

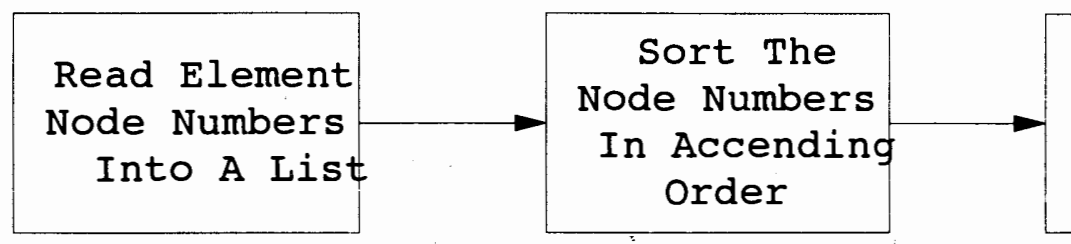

Delete Duplicate Node Numbers

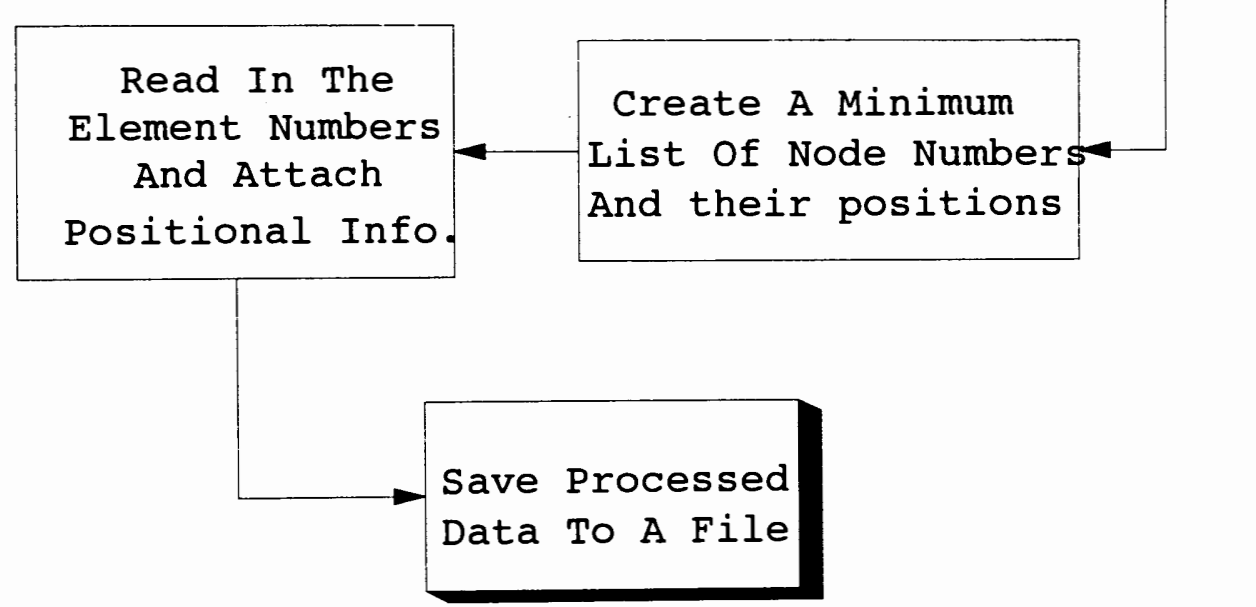

Figure 4 - Flow chart of algorithm for processing tool mesh data. 
The element information consisted of nine columns, and as many rows as there were elements in the mesh around the tip of the tool. The first column contained the element numbers, and the remaining eight consisted of the node numbers of the respective element, please refer to Figure 5.

$\begin{array}{ccccccccc}\text { E\# } & \mathrm{N} 1 & \mathrm{~N} 2 & \mathrm{~N} 3 & \mathrm{~N} 4 & \mathrm{~N} 5 & \mathrm{~N} 6 & \mathrm{~N} 7 & \mathrm{~N} 8 \\ 325 & 546 & 562 & 565 & 551 & 621 & 647 & 755 & 691 \\ 329 & 551 & 565 & 568 & 550 & 691 & 755 & 762 & 698 \\ 333 & 550 & 568 & 571 & 549 & 698 & 762 & 769 & 705 \\ 337 & 549 & 571 & 574 & 548 & 705 & 769 & 776 & 712 \\ 341 & 548 & 574 & 577 & 547 & 712 & 776 & 783 & 719 \\ 345 & 547 & 577 & 555 & 545 & 719 & 783 & 670 & 663 \\ 493 & 615 & 629 & 761 & 685 & 580 & 582 & 600 & 599 \\ 497 & 685 & 761 & 768 & 692 & 599 & 600 & 601 & 598 \\ 501 & 692 & 768 & 775 & 699 & 598 & 601 & 602 & 597 \\ 505 & 699 & 775 & 782 & 706 & 597 & 602 & 603 & 596\end{array}$

Figure 5 - Sample of ANSYS generated element node data.

The second set of data contained the node positions. The node position data generated by ANSYS was formatted in four columns and as many rows as there were nodes in the mesh. Figure 6 shows a sample of the node data. The first column of the nodal data contains the node numbers, with the remaining three columns being positions in $\mathrm{x}, \mathrm{y}$ and $\mathrm{z}$. The coordinates of the nodal positions are in "nodal space," which is determined when the tool is constructed and meshed in ANSYS. 


$\begin{array}{cccr}\mathrm{N} \# & \mathrm{X} & \mathrm{Y} & \mathrm{Z} \\ 545 & 20.000 & 23.000 & 10.000 \\ 546 & 20.000 & 18.000 & 10.000 \\ 547 & 20.000 & 22.167 & 10.000 \\ 548 & 20.000 & 21.333 & 10.000 \\ 549 & 20.000 & 20.500 & 10.000 \\ 550 & 20.000 & 19.667 & 10.000 \\ 551 & 20.000 & 18.833 & 10.000 \\ 552 & 29.000 & 23.000 & 10.000 \\ 553 & 26.346 & 23.000 & 10.000 \\ 554 & 23.982 & 23.000 & 10.000\end{array}$

Figure 6 - Sample of ANSYS generated nodal position data.

Not all of the elements of the tool mesh were reconstructed as solids. Only those elements in the mesh that were in close proximity to the cutting edge were reconstructed as solids. By using a subset of the elements, the number of computations per time-step was reduced. The subset of the elements to be built as solids were described in the element data file, which was generated from the FEA package. While the element file was a subset of the elements that described the tool, the node position file contained the complete set of nodal information. Because of this fact, the node file contained more information than was needed to reconstruct the elements as solids. For this reason, the process of converting the data began by extracting the minimal set of node numbers from the element data file, so that a minimal set of nodal positions could then be extracted from the node data file. 
The minimum set of node numbers needed for the subset of elements was obtained by reading all of the node numbers from the element file, sorting them and removing the duplicate values. In Scheme. the data was represented as a single level list of numbers in acceding order. From the node file, the positions for the minimal set of nodes, were read in and stored in a two level list. The top level of the list was made up of pairs which contained a node number and a position entity. The form of a 3DToolkit position entity is: \#[position $\mathrm{x} \mathrm{y} \mathrm{z}$ ]. The minimal list of node numbers and positions had the form: ((node_number \#[position x y z]) (node_...) ...).

The node position list and the element node list were then used to generate a file which contained the element number, followed by the eight positions of the element's nodes. The final format of the mesh data consisted of a group of nine lines, with the groups separated by a blank line. Each of the groups contained the data for a single element. The first line of the group is the element number, which is followed by eight lines of node positions in $x, y$, and $z$. An example of one element's information is shown in Figure 7. 
201810

21.8761810

21.87618 .83310

2018.83310

201810.25

21.8761810 .25

21.87618 .83310 .25

2018.83310 .25

Figure 7 - Example of Processed Element Data.

At this point in the process, the node position data has yet to be transformed into an order that the solid modeling software can use. The node numbers themselves are preserved by the order of the positional information. A reference to the element data file must be made to determine a node number. In this manner, a minimal set of data is used to generate the finite elements as solids. The element data file is referenced for the node numbers when the cutting force information is written to a final file that is formatted so the force data can be imported into ANSYS.

\subsection{Elements as Solids}

The algorithm used to generate the finite elements as solids is represented in the flow chart referred to as Figure 8 . 


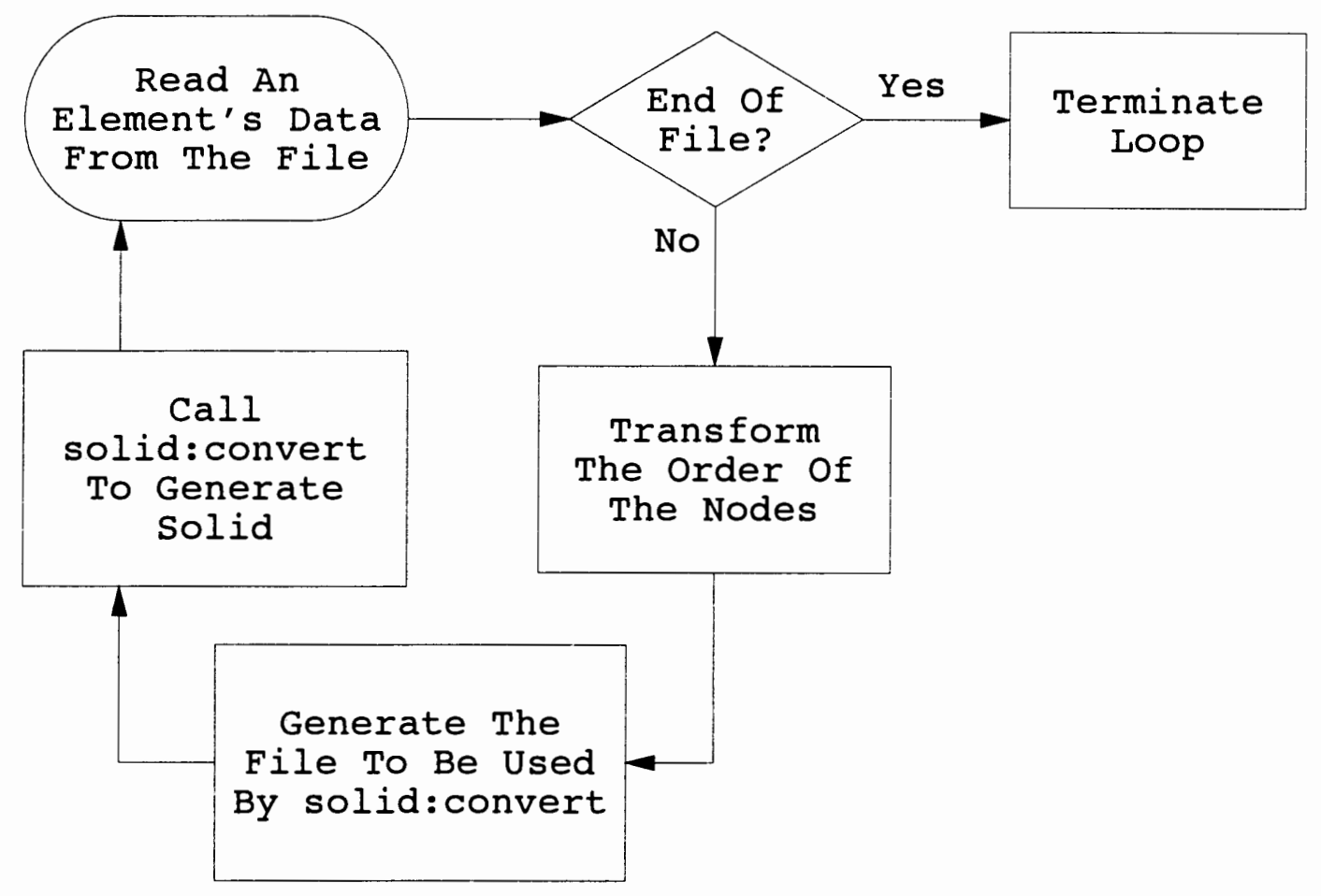

Figure 8 - Flow chart of the algorithm to process the tool mesh data to produce a version of the tooi made of solid bodies in the shape of the elements.

The function used to build the solid elements was an extension to the 3DToolkit named "solid: convert". Solid: convert required the positional information of an element in a different order than was generated by ANSYS. The transformation of the nodal information was determined by observing the order of the node numbers that the ANSYS data was written in and the given order that solid: convert required to construct solids. From these observations, a 
transformation of the order of the node numbers was determined and implemented as a Scheme routine. Figure 9 displays the transformation applied to the vertex list.

\begin{tabular}{cc} 
Before & After \\
\hline a & f \\
b & g \\
c & h \\
d & e \\
e & a \\
f & d \\
g & c \\
h & b
\end{tabular}

Figure 9 - An example of a list before and after the transformation.

The method of execution used to transform the positional information was to convert a list of positions to a vector, which is a Scheme data type. The items of a vector can be easily referenced by their position in the vector. The transformation was executed by copying the items in the old vector to their transformed position in the new vector. This new vector was then converted to a list data type.

Because solid: convert was not part of the 3 DToolkit, its usage was undocumented and had to determined by experimentation. The solid: convert routine had the following three requirements and restrictions: its input data had to be written to a formatted file, attributes of type real could not be attached to solid bodies 
created with it, and a working coordinate system (WCS) had to be defined in model space prior to its use.

The input data for the solid: convert routine had to be written to a formatted data file prior to being used for the generation of an element. The data input requirement motivated the need for an iterative approach in which the information was written to a file in the specified format, one element was generated, the next element's information was written to a file, and so on. The file had to be stored in the same directory that the executable for the 3DToolkit was located, and it's name ("TEST2") was hard coded into the 3DToolkt executable. The modified executable was stored under a directory named "facet" in order to distinguish it from the unmodified version of the 3DToolkit. The format of the data file used by solid: convert was as follows:

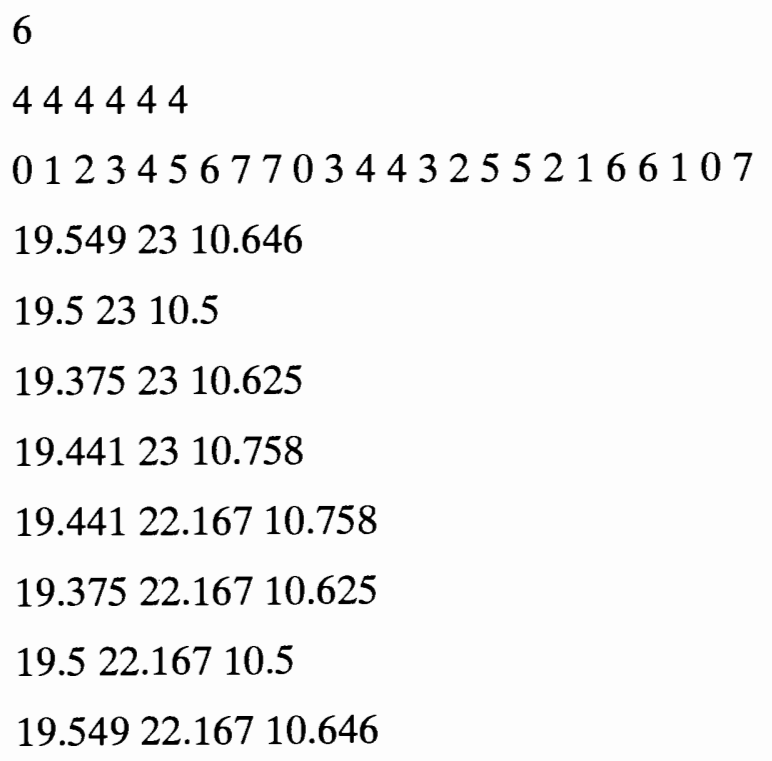

Figure 10 - Format of the data file used by the solid:convert routine. 
The first line of the file used by solid:convert specified the number of faces of the solid, the second line designated the number of edges per face, the third line set the vertex list of the six faces. It most likely would have been possible to change the order of the vertex list to match the order that the nodal data that was generated by ANSYS. By doing this, it may be possible to eliminate the need for transforming the order of the data, as described above. The need to convert elements of other types, than eight node bricks, needs to be taken into consideration when modifying the vertex list. For this reason, the method of applying a transformation to the node order was implemented. The remaining eight lines of the file contained the position information of the vertices, in $\mathrm{x}, \mathrm{y}$, and $\mathrm{z}$ and in the transformed order suitable for the above vertex list. The first three lines of the file were unchanged for all of the elements constructed in this experiment. These three lines would change if a different type of finite element, such as a tetrahedral, were being constructed. The code developed to do this was left open for optional element types. The position information changed with the generation of every element.

After an element was constructed, an attribute was attached to the entity which had the element number as its name. Attributes are 3DToolkit data types which can be attached to an entity and are saved and restored with an ACIS part file. During the process of determining the magnitude of the forces, attributes were used as a means of managing this information. 
The second requirement of using the solid:convert routine was that entities generated by solid: convert could not have attributes attached to them which had values of type real. An attribute is made from a dotted pair, which is a Scheme data type, with the following format: (item 1 . item2). The first item of an attribute is a string which represented the name of the attribute. The second item in the dotted pair is the value of the attribute. An attribute value is, in general, able to be of type string, real, integer, Boolean, position, or gvector. Attributes with values of type real could not be attached to an entity that had been constructed using the solid: convert routine. For this reason, the value of the attributes were attached to the elements as strings. By naming the entity with its ANSYS generated number at the time of it construction, it was easily identifiable when the time came to determine the forces on the element's nodes. Identification of the nodes was accomplished by the fact that the order in which the vertices were returned by the $3 D$ Toolkit was the same order as used by the solid: convert routine to construct the element as a solid.

The third requirement of using the solid convert routine was the need for a working coordinate system to have been defined in model space prior to calling solid: convert. A working coordinate system is an entity in the solid modeling environment. The solution to this requirement was easy to implement but not initially apparent. Prior to the construction of the solids in model space a working coordinate system was created at the origin of model space. After all of the elements were created, 
a working coordinate system was also created in the same location on the multi-lump version of the cutting tool as one that had been placed on the one lump version of the tool. This later working coordinate system was used to align the chips with the meshed version of the tool, in the same orientation that they were generated in with the one lump version of the cutting tool.

\subsection{Limitations}

The code generated in the development of this algorithm handled only eight node brick elements. The limitation of eight node brick elements was imposed in an effort to simplify the problem. The code was constructed so that future element types could be handled by inserting the proper vertex list transformations into the routine which generates the file called by solid: convert. The routines which manipulate the two original data files, to produce a single data file, will have to be modified to account for elements other than eight node bricks. 


\section{Modeling A Cutting Process}

\subsection{Introduction}

The models which were constructed during the development of the algorithm, were done so in the 3DToolkit solid modeling software package from Spatial Technologies. The construction of a model begins with a geometric description of the cutting tool and the stock to be cut. The tool is oriented in space in a position from which the start of the cutting process occurs. The starting position is referred to as the datum position. The transformations, which specify the relative path that the tool will follow during the cutting process, are also defined in the $3 D T$ Tolkit. For both of the models developed here, the stock is held in a constant orientation, and the transformations are applied to the tool only. The magnitude of the transformations are defined to obtain the desired resolution of the cutting process. Applying one transformation to the tool, from the datum position, causes an intersection of the tool and work piece to be true. Running the model involves applying the transformations to the tool and work piece, while performing Boolean intersection and subtraction operations on the solid bodies. This is done in an iterative manner. The result of each iteration is a "chip". Several iterations performed in sequence produce a set of chips. Each chip discretely describes the geometric interaction that occurred between the cutting tool and work piece. The chips are grouped in batches of several chips, and saved to a part file for later use in the approximation of the cutting forces. The 
orientation, in model space, that the chips were in when they were created is preserved when they are saved to the part file. The orientation of the chips is used in the determination of the cutting forces to approximate the cutting force directions.

This method of recording the interaction between a cutting tool and work piece represents a discrete history of the cutting process in the form of the chips. The overall algorithm for modeling a cutting process is shown in Figure 11. 


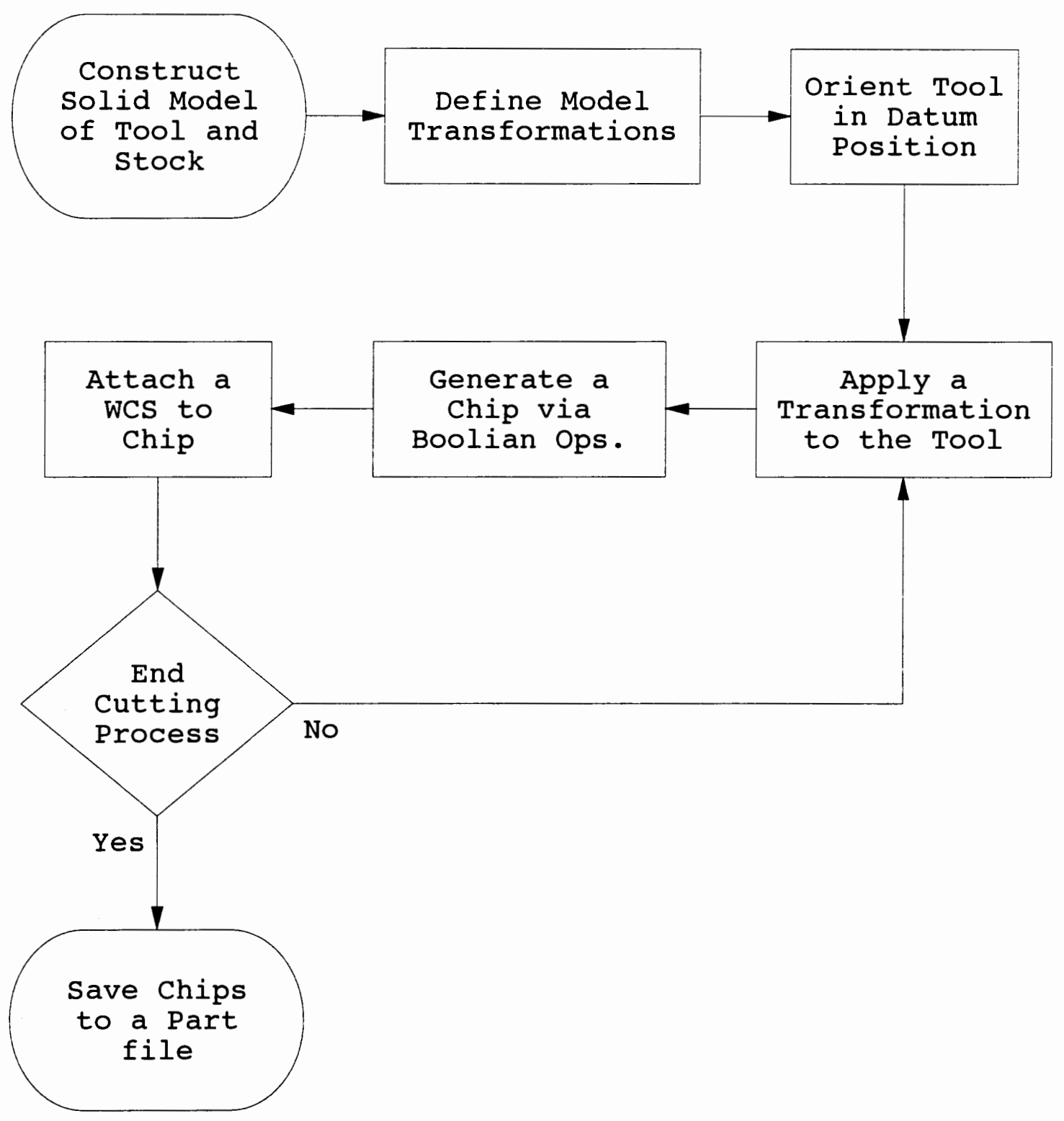

Figure 11 - Flow chart of the algorithm for modeling a cutting process.

The end of the cutting process is determined by how many chips are to be generated, and is set by the user. Models are as individual as the cutting process, and consume a large percent of the time required to set up this analysis. The environment 
in which the models are constructed is based on a Scheme interpreter. This programming environment provides the modeler with all of the power of a high level programming language for the development of models but tends to make the task of constructing a model a tedious process.

\subsection{Tool Construction}

As in the physical world, where a single tool can be used for several different cutting processes, a single tool can be used for several models of cutting processes in the computational space of a solid modeling environment. In both of the models discussed in this work the same tool is used. There are at least three general approaches that can be used to construct a solid model of a cutting tool. The first method involves using solid modeling functions provided by the 3DToolkit to construct the tool, and was the method utilized to develop the tool used in these models. As the tools become more complex in shape this method will not be very practical. The command line nature of the Scheme interpreter makes it difficult to build complex shapes in a reasonable amount of time. A better method of building a single lump tool will be to unite a copy of the lumps created from the meshed tool data, which was generated by the finite element package. A third approach to constructing models of tools, would be to use another solid modeling software package, such as Trispective by $3 D$ Eye or Pro/Engineer by PTC, to construct the solid model of the cutting tool. Software packages such as these provide a high degree of 
functionality for generating complicated shapes. The model of the tool would then be imported into the $3 D$ Toolkit, for use by the routines that determine the cutting forces, as well as imported into the finite element software for meshing.

The tool used in these models was a simple shape, consisting of a rectangular block with two edges chamfered at one end to form a fifth edge. The tool was symmetric about a center line along it's length, and is shown in Figure 12.

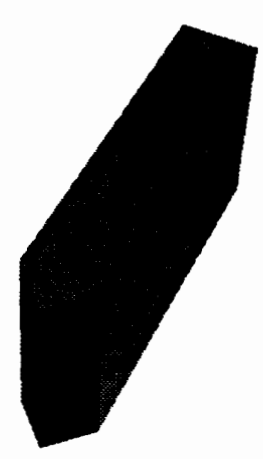

Figure 12 - Single lump solid model of the cutting tool.

An important aspect to be considered when constructing a model of a cutting tool is providing an easy means of aligning the cutting tool with the chips created from 
the modeling process. The alignment of the chips with the tool takes place when the magnitudes of the forces are determined. The chips are transformed to nodal space and aligned with the multi-lump version of the cutting tool. The alignment at that later point in the algorithm was accomplished by creating a working coordinate system (WCS) entity on the single lump version of the cutting tool and attaching a copy of that WCS to each chip. A WCS was created at the cutting end of both tools in the same relative location. The WCS was placed by determining an extreme box about the tool lump(s) and using one of the vertices at the cutting end of the extreme box. This method of placing a WCS for alignment will be applicable to more complex shaped cutting tools as well as this simple symmetric tool. The WCS is attached to the chip while the tool is in the orientation that the respective chip is being created. When the forces are calculated, each chip is aligned with the meshed version of the tool by translating the WCS of the chip onto the WCS of the tool and rotating the chip so that it is properly aligned with the tool in nodal space. Only cne set of transformations need be defined to do this, as they are applied to all of the chips from the datum position.

After the tool has been created, it is saved to a part file, from which it can be restored into model space at any time. All parts are restored into model space in the same location and orientation in which they were created. 


\subsection{Transformations}

From the position a tool is restored from a file into model space, transformations are applied to the tool in order to place it in the datum position. In the 3DToolkit, transformations are objects which are used to manipulate the position of top-level entities, such as the model of a cutting tool, in model space. As well as placing the tool in the datum position, transformations were used to implement the movement of the tool along it's path that modeled the cutting process. The 3DToolkit contained a variety of routines which could be used to transform entities. Transforms are very powerful entities for model construction that can be combined and bound to variables for reference by name. After a transformation had been defined, it can be called in an iterative manner. The inverse of the transformations defined for the model of the cutting process were used to untransform the chips and align them with the datum position.

Some transforms are simple to create, such as the following translation of the tool, in the $\mathrm{x}$ direction, by the value bound to the variable carriage-ss (carriage step size).

(entity:transform tool (transform:translation (gvector carriage-ss 00$)$ ))

WCS to WCS transformations provide a simple means of moving objects in model space. When the tool was restored from the part file, the first transformation 
applied to the tool was a WCS to WCS which piaced the tool in an area of model space designated as the "tool-post". The following transformation is an example:

$$
\text { (entity:transform tool (wcs:to-model-transform tool-post) ) }
$$

From the tool rest, transformations were defined which, step by step, placed the tool in the desired starting, or datum position. These transformations were more difficult to implement, because the positions in space for which they were to be applied were not as easy to find as the position of a WCS is. The following is an example of a transformation that moves the tool from a working coordinate system to the end of the work piece where the tip of the tool just touches the stock:

\author{
(entity:transform tool \\ (transform:translation \\ (gvector:from-to tool-tip \\ (point:position cut-point))))
}

In this transformation, the tool is translated along a geometric vector from and to previously determined points on the tool and work piece respectively. This transformation was used in model two, which models the cutting process of a lathe. In model two, the tool in the datum position was oriented with the cutting depth applied to the tool and the tool tip on the center line of the cylindrical stock. The tool had its cutting edge just touching the edge of the stock. Placing the tool in this datum position 
required the use of rays to determine the position of the edge of the tool. As tool geometry becomes more complex, the use of rays will provide the means to orient them in the datum position.

Both models used one set of transformations to specify the path of the tool, so that the path of the cutting process was constant. By applying the transformations to the tool only, all of the information needed to determine the direction of the cutting force was preserved in the orientation of the chips in model space. The chips were save and restored to and from a part file. When the chips are restored from the part file to calculate the forces, the direction of the cutting force is determined as the inverse of the transformations used to run the model are applied to orient the chips in the datum position.

A difficulty with the routine provided for transformations of geometric vectors (gvectors) should be noted here. Through experimentation it was discovered that the function "gvector:transform" did not work in the manner in which it was documented. The documentation stated that the routine operated in the same manner as those provided to transform of other entities, such as solid bodies and WCS's. The functions other than gvector:transform apply the transformation to the entity, gvector transform returns the transformed gvector, but does not update the direction of the gvector. In order to apply the transformation to a gvector, the "set!" command must be used, to bind the variable name to the value returned by the function "gvector:transform". 


\subsection{Model One}

In the first model, the tool was rotated about a vertical axis which was midlength of the tool in the plane of the centerline and normal to the top surface of the tool. The front end of the cutting tool passed through a square block as the tool is rotated about the axis. The tool was rotated at a constant angular step size of 4 degrees, between which Boolean operations were performed to generate the chips and update the shape of the work piece. The result of this cut was to remove a half moon shaped volume from the block. The starting position of the tool was at angle of 50 degrees to the face of the block. The position of the tool at this point was defined as the datum. The tool was advanced through the stock for a total sweep angle of 80 degrees. The model, close to the end of its sweep, is shown in Figure 13. 


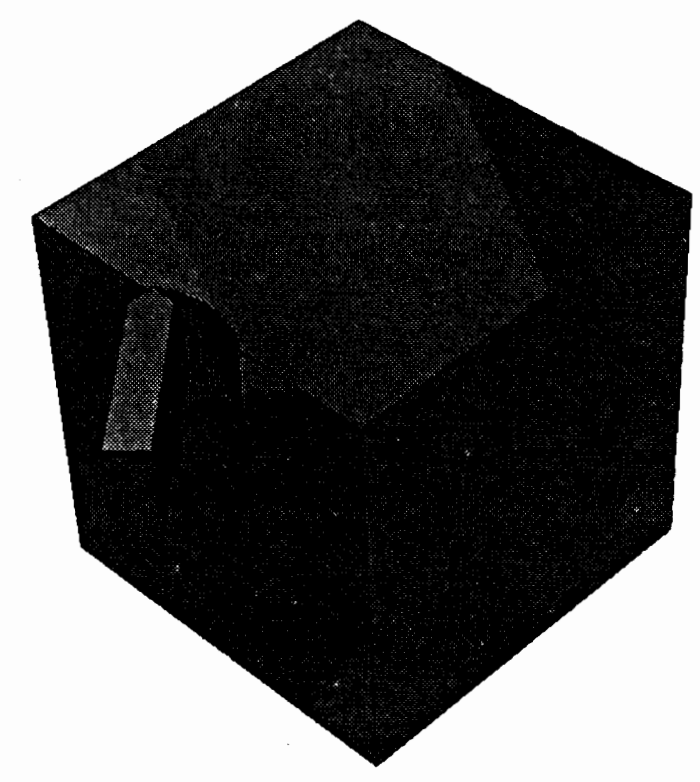

Figure 13 - Rendered image of Model One.

The chips were saved to a part file in their final form, which is shown in Figure 14.

The WCS attached to each chip was also saved to the part file and is not shown. 


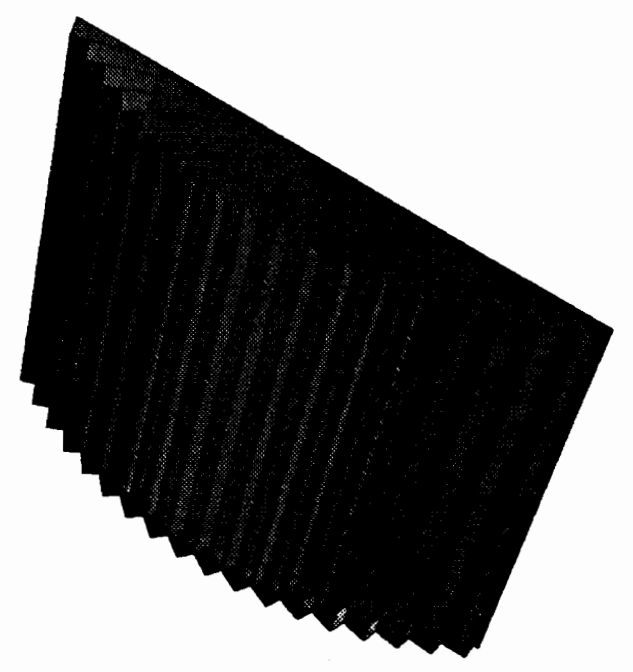

Figure 14 - Model One chip set.

\subsection{Model Two}

The second model consisted of the same cutting tool that was used in the first model. The work piece in the second model is a solid cylinder. The transformations in the second model were developed to resemble the cutting process of a lathe. An actual lathe rotates the stock and translates the tool to implement the cutting process. The transformations of this model were implemented by rotating and translating the tool about and along the axis of the stock. Keeping the stock stationary and rotating the tool does not reflect the actual workings of a lathe, but the relative path of the cutting tool is the same, although discrete in nature, as that of a lathe. The datum position of the cutting tool was defined at the right end of the stock with the end of the tool advanced 
to the cutting depth and the left cutting edge of the tool making contact with a point on the stock.

There are two reasons for rotating the tool only. The first of which was computational efficiency. As the model of the machining process proceeds, the complexity in the shape of the stock increases. This increase in complexity of the stock is directly proportional to the increasing number of subtractions that occur between the tool and work piece. The added complexity of the work piece makes translating or rotating that work piece more and more computationally expensive as the process proceeds. While the shape of the stock changes continuously, throughout the cutting process, the shape of the tool remains the same. For this reason, it is more computationally efficient to transform the tool only. No chipping of the tool was taken into consideration in this work. The second advantage to translating just the tool, is that the path of the tool is preserved by the orientation of the chips in space. The direction of the cutting force was defined by the geometric vector created from the centroid of a chip in one orientation to the centroid of the same chip after having had the inverse of the transformations which define the path of the tool, applied to it one time. Figure 15 shows an image of model two after the tool has completed four revolutions around the stock. 


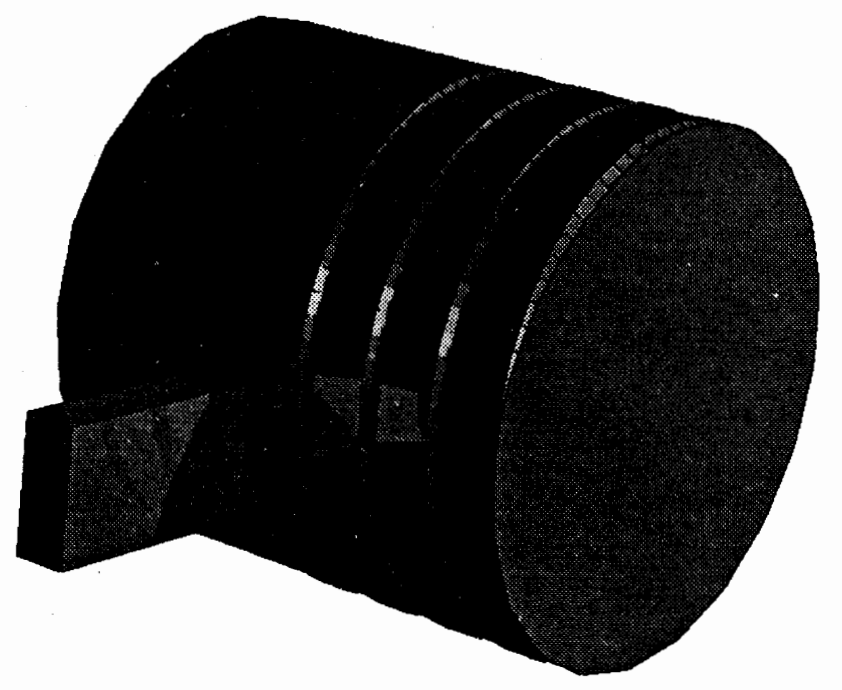

Figure 15 - Rendered image of Model Two.

As can be seen from a rendered image of the chips in Figure 16, the path of the tool is preserved by the orientation of the chips in model space. 


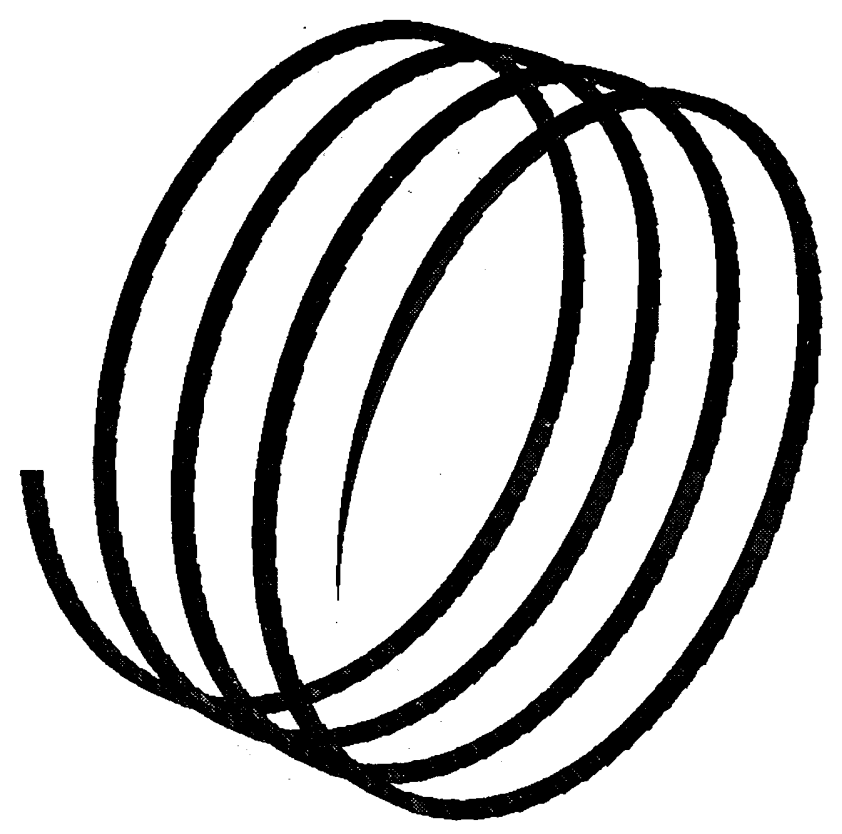

Figure 16 - Model Two chip set.

\subsection{Chip Generation.}

The Boolean functions which are part of the 3DToolkit are destructive operations. When two solids are passed to a Boolean function to determine their intersection, both of the original solids are destroyed (the entities are deleted from the environment) and the entity resulting from the operation is bound to the first argument 
passed to the function. Because of the destructive nature of the Boolean operations, copies of both the tool and work piece are used to generate the chips. Having generated a chip, the WCS of the tool is attached to the entity list of the chip, and the shape of the stock is updated to reflect the removal of material. The following algorithm is performed in an iterative manner to model the cutting process. The operations below are performed after the tool has been oriented in the datum position. Each description of the operation is followed by the Scheme code used to perform it. This example comes from the program that defines model two.

- Make a copy of the stock prior to the cut. (set! stock-temp (entity:copy stock))

- Translate the tool, by the user defined value of the carriage step size. (entity:transform tool (transform:translation (gvector carriage-ss 00 )))

- Rotate the tool around the stock, by the user defined value that is bound to "rotation". (entity:transform tool (transform:rotation (position 0 10 0) (gvector -1 0 0) rotation))

- Make a copy of the tool in its new position where it is engaged with the stock. (set!' tool-temp (entity:copy tool))

- Perform a Boolean intersection of the tool copy and the copy of the stock. The result of this operation is the "chip", which is bound to the variable "stock-temp". 
The entity list of the tool is made up of the WCS and then the lump. For this reason a "cadr" operation is performed on the entity list returned by "tool-temp". (solid:intersect stock-temp (cadr tool-temp))

- Attach the tool's WCS to the chip, in the current orientation of the tool. (set! stock-temp (append (list (car tool)) (list stock-temp)))

- Copy the chip's solid body and WCS to the part "chip-set", so that it can be saved to a file containing only the chips generated by the model. (entity:copy stock-temp chip-set)

- Make a copy of the tool for the purpose of updating the shape of the stock. (set! tool-temp (entity:copy tool))

- Perform a Boolean subtraction of the tool copy and the stock, to update the stock's shape.

(solid:subtract stock (cadr tool-temp))

- Go to the beginning.

The result of executing a model of a cutting process, is a set of chips. The chips are saved to a part file, from which they can be restored to model space. The chips, and the inverse of the transformations used to create them, are utilized in the force calculation algorithm to approximate the forces acting on the nodes of a finite element mesh. 


\section{Force Calculation Algorithm}

\subsection{Introduction}

The forces that are calculated by this algorithm are derived from the three dimensional geometry of a solid model of a "chip". A chip is defined here as the intersection of a cutting tool and work piece at a discrete instance in time. The chip geometry is the result of modeling a cutting process in computer software. Models are executed and produce a discrete history of a cutting process in the form of a set of chips. The result of applying the force algorithm to a single chip are the components of the cutting forces, in three dimensions, acting on the tool at the nodal level, during an instant in time. The nodal cutting forces are simply proportional to the volume of intersection of each the elements with a chip. Two simple models were used to develop the algorithm for determining the cutting forces. One tool shape was used in both of the models. The version of the tool used to calculate the forces consisted of a set of solid elements which were equivalent to those of the finite element mesh generated by a finite element software package. This meshed version of the cutting tool had the same dimensions as the single lump version of the tool, used to create the chip volumes. In order to facilitate the implementation of this approach of determining the cutting forces, a simple method for approximating both the direction and magnitude of the cutting force was developed. The methods developed were 
implemented using the solid modeling software package ACIS 3DToolkit from Spatial Technologies Inc..

The direction of the cutting force was determined by applying the inverse of the transformations, used to generate the chips in the modeling process, to align the chips with a datum position. The datum position of the cutting process was defined as the position of the tool at the start of the cutting process, prior to the application of any of the tool path transformations to the tool. The direction of the cutting force was determined for each chip by applying the inverse transformations to the chip, until it was one time-step away from being aligned with the datum position. At this orientation of the chip in space, the centroid of the volume was computed. One final transformation was then applied to the chip so that it was oriented in the datum position, and the centroid of the chip was again determined. The direction of the cutting force was defined as a geometric vector from the position of the centroid one time step prior to the datum, to the position of the centroid of the chip oriented at the datum. The force direction vector could be determined between any two time-steps in which the transformations were constant. By determining the direction vector at the datum, only a single set of transformations need be defined to orient all of the chip's direction vectors with nodal space. Nodal space is defined by the position and orientation of the meshed version of the cutting tool, which is created in the finite element package. It was important to determine the cutting forces in nodal space so that they could be properly applied to the tool in the finite element software package. 
The magnitudes of the node forces, determined by this algorithm, were derived from the three dimensional geometry of the chip. The forcing algorithm processes each chip to determine the amount of force being applied to each of the nodes of the finite element mesh. Each chip represents a discrete point of the cutting process. In order to calculate the magnitude of the force acting on a tool at a point in time, a chip is aligned with the meshed version of the cutting tool. The meshed version of the tool differs from the single lump version of the tool used to create the chips in two ways. The first difference is that it is made up of many separate solids which have the same shape as the finite elements. The second difference between the meshed tool and the single solid lump version, is that only the portion of the tool in the vicinity of the cutting edge of the meshed tool exists. After having aligned a chip with the meshed tool, each of the elements of the meshed tool were intersected with the chip. If an intersection existed then the volume of intersection became a subchip. For each element of the mesh that had a subchip, the magnitudes of the forces acting on the nodes of the element were determined, based on the size of the subchip and its orientation with respect to the element.

The magnitude of a force acting on a single node of an element was determined by dividing the volume of the subchip by the distance from the node to the centroid of the subchip. This magnitude for each node was normalized by multiplying it by the sum of the distances of each of the nodes of an element, to the centroid of the subchip. 
In this manner, the sum of the magnitudes of the forces acting on the nodes of a single element was equal to the volume of the subchip. A single time-step was processed when each of the elements had been intersected with the chip. After processing a chip, the nodes which had adjacent elements had their magnitudes summed. The final step in producing the results, was to apply the magnitude to the unitized direction vector and store the information in a file.

The process of keeping track of the nodal force data was facilitated by the use of attributes. An attribute is a general purpose data entity that is part of the 3DToolkit. Attributes can be attached to other entities in the 3DToolkit and are save and restored as part of a model. Each of the element lumps had attached to it a list of attributes. The data attached to the element solid bodies, included the element number, the node numbers of that element, and each node's respective force magnitude.

\subsection{The Direction of the Cutting Force}

The chips generated from the execution of a model are orientated in model space in the position in which they were created during the execution of the model. As an example of chip orientation, the results of executing model two are shown in Figure 17. The execution of model two produced a set of chips arranged in a helix. 


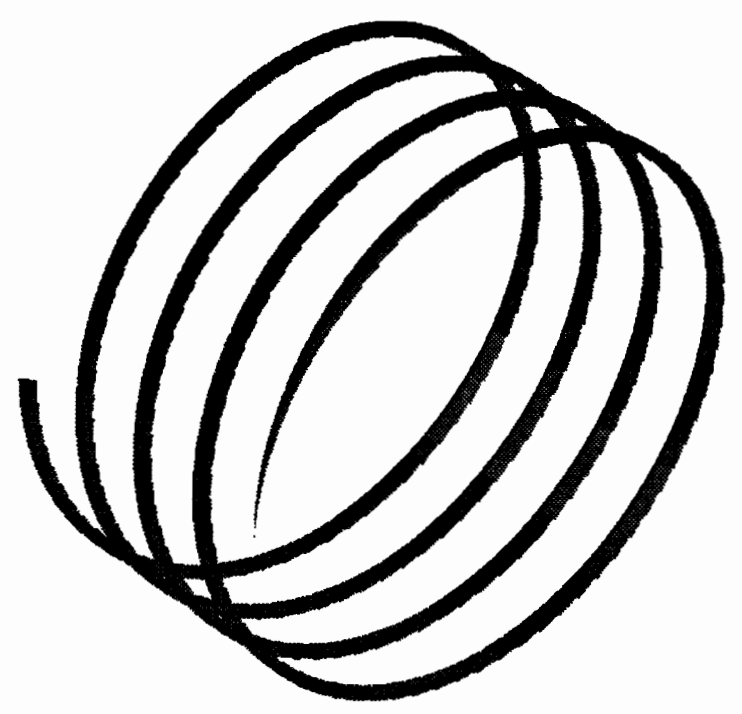

Figure 17 - Model Two chip set.

Because the transformations which define the path were applied to the tool only, the information of the path was preserved in the orientation of the chips in space. This preservation of the path information allowed for the determination of the direction of the forces in the section of the algorithm where the magnitudes of the cutting forces were determined. The direction of the force, or direction vector, was approximated by applying the inverse of the transformations, that were applied to the tool when the machining process was modeled, to each chip being processed in the 
calculation of the cutting forces. This method of determining the direction of the cutting force applies to a cutting process in which the transformations that describe the path are constant between time-steps. We will call a cutting process of this type "simple". A complex cutting process can then be defined as one in which the transformations which define the path are not necessarily constant between time-steps. A change in the transformations between time-steps would indicate the end of one simple cutting process, and the beginning of another. By defining simple cutting processes in this manner, a complex cutting process can be reduced to the sum of several simple ones. The tool paths considered in the models described here are all of the simple type.

For each of the two cutting processes modeled, a datum position was defined at the beginning of the cutting process. Defining the datum at this position allowed the inverse of the transformations used to model the cutting process, to be used to align the individual chips in the datum position. From this datum position it was possible to define a single set of transformations which would align each individual chip, and its respective direction vector, with the meshed tool in nodal space. Nodal space was determined by the position and orientation in which the tool was constructed and meshed in the finite element software package.

The direction vector was defined as the geometric vector taken from the centroid of the chip in an orientation which was one tool path transformation from the 
datum position, to the position of the chip when oriented at the datum position. After having determined the direction vector in this manner, a set of rotational transformations were applied to the vector to orient it with nodal space. The set of transformations used to orient the direction vector with nodal space were the same as those used to orient the chips with nodal space after they had be translated to nodal space. The direction vector was used to determine the components of the force for all of the nodes of the tool mesh at the instant in the cutting process represented by the chip. The calculation of the direction vector differed from the calculation of the magnitude in that it was done at the chip level, where the magnitudes were calculated at the subchip, or element level. The same direction vector was applied to all of the force magnitudes calculated for a single time-step.

The direction vector was attached to the chip entity as in attribute. This allowed the direction vector to be saved and restored with an ACIS ".sat" part file. Saving the data to a part file at this point in the process was not required, because the information is processed to completion while in memory. This does provide another point in the algorithm that could be taken advantage of to break the code into smaller segments. The calculation of the cutting forces requires more system resources to process than any other part of the algorithm developed to this point. 


\subsection{The Magnitude of the Cutting Force}

This calculation of the magnitude of the cutting force is based on the volume of intersection of the tool and work piece at an instant of time. The magnitude of the force acting on a single node of the mesh is proportional to the volume of intersection of a subchip, and the inverse of the distance of the node to the centroid of the subchip. The greater the distance of the node to the centroid of subchip, the smaller the magnitude of the force on the node. In order to normalize the forces acting on the nodes of an element, the sum of the nodal weights for a single element, is defined to be equal to the volume of the chip and element intersection (volume of the subchip).

$$
\sum_{n=1}^{8} W_{n}=v o l
$$

This formula above applies to eight node elements, where $W_{n}$ is the magnitude or weight of the force acting on a single node, and "vol" is the volume of intersection of the chip and the current element. The nodal magnitudes are normalized by multiplying each of the nodal weights by the sum of the distances of the individual nodes to the centroid of the subchip. The normalization is expressed in the following equation for the magnitude of the force acting on a single node: 


$$
W_{n}=\frac{\sum_{m=1}^{8} d_{m} * v o l}{d_{n}}
$$

For a single node, the magnitude of the force is equal to the volume of the subchip divided by the distance of the node to the centroid of the subchip, times the sum of the distances of all the nodes of the element to the centroid of the subchip.

\subsection{Algorithmic Details of Force Calculations}

After the chips and meshed version of the cutting tool are loaded into model space, if it is a new model, then a set of transformations are determined and coded to align the chips with the meshed version of the tool in nodal space. Once the model specific nodal alignment transformations have been developed, the remainder of the coded algorithm can be implemented unchanged. For each chip in the chip list, the first WCS and solid body is extracted from the list of chips. The chip list is then updated to reflect this change. The inverse of the transformations used to create the chip is then applied to the current chip. When the chip is one time-step from being oriented in the datum position, the centroid of the chip volume is determined, in model space. The final inverse transformation is applied to the chip, and the centroid of the chip is again determined, in model space. From these two positions, a geometric vector is defined and unitized. This same rotational transformations used to align the chip 
with the meshed tool in nodal space are applied to the geometric vector, so that it is oriented properly in nodal space.

The next step in determining the nodal forces is to intersect the current chip with each of the elements, to create subchips. If a solid body is created when the chip and element are intersected then the volume of the subchip is calculated and it's centroid found. Once the centroid of the volume of the subchip is found, its location is used to find the distance to each of the eight nodes. Upon compiling this information, it is used in the equations, described above, to calculate the magnitudes of the forces acting on each of the nodes. The weights for each node are attached to an element as attributes. In order to make the calculation of the summing of the weights for nodes with adjacent elements mode efficient, a list of the element entities that have an intersection with the chip is kept. When there are no more elements to be intersected with the chip, then the processing of the chip with the elements is done. For each node that has several weights calculated from adjacent elements, the weights are summed. The nodal weights are multiplied by a user defined scalar, which has been kept at unity up to this point in the development of the process. The scalar was implemented as a possible way of dealing with issues that are unaccounted for at this point, such as material properties. The final step in determining the nodal forces is to combine the direction vector and magnitude information and save it to a file in a format that the finite element analysis software can access. An example of the formatted force data is shown in Figure 18. 


$$
\begin{aligned}
& \text { F,860,FX, } 0.0292808483590014 \\
& \text { F,860,FY, 1.02233594844623e-016 } \\
& \text { F,860,FZ, -0.189570580604961 } \\
& \text { F,864,FX, } 0.00500631368768003 \\
& \text { F,864,FY, } 1.74794609410294 \mathrm{e}-017 \\
& \text { F,864,FZ, - } 0.0324119636435436
\end{aligned}
$$

Figure 18 - Sample of final output of the calculated cutting forces.

Where the first field dictates that the data is a force, the second field contains the node number for which the force applies, the third field specifies the direction of the forcing component in nodal space and the final field is the magnitude of the force component in nodal space.

Prior to processing the next chip, if one exists, all of the relevant variables are reinitialized and the entities that have been processed in the current iteration are deleted.

\subsection{Storage of information as attributes}

The element number, which was assigned to the element when the mesh was generated in ANSYS, was attached to the element when it was constructed as a solid. In order to simplify the coding of the algorithm, attributes were used to manage the weighting information as is was being calculated. An attribute is a general purpose 
data type, defined by the 3DToolkit, that is used to attach information to entities. Attributes are attached to an entity as a list of dotted pairs. A dotted pair is a Scheme data type, which has the following form: (atom1 . atom2) A pair of atoms are placed in a list, bounded by parenthesis and separated by a period. The first atom of an attribute is a string, which is the attribute's name. The second atom of the attribute is the value, which can be a string, real, integer, position, or Boolean. The use of attributes to manage the information calculated when the forces are determined had two advantages. The first advantage being that it eliminated the need to create and manage separate lists of information. The 3DToolkit provided several handy functions for accessing, adding, removing, or replacing attributes. Several attributes could be attached to a single entity, and a single attribute could be easily attached to several entities. These properties of attributes made them very useful for handling the data generated when the cutting forces are calculated. The second argument for the use of attributes is that they are saved and restored with the part file. This factor provides a means of being able to break the forcing algorithm into smaller pieces, should it be required in the future.

Version 1.2 of the 3DToolkit was used to write all of the code that this thesis is based on. The build of the 3DToolkit, was extended to include the function called "solid: convert", which was used to create the elements if the mesh as solid bodies. For reasons unknown, it was only possible to attach attributes to the solid bodies created with the solid: convert function that had values of type string. For 
this reason the values of the nodal weights were converted to strings before being attached to the element lumps. 


\section{Results}

\subsection{Introduction}

The result of this work is an algorithm that partially completes the process of computationally determining the condition of cutting tools. The process is developed to the point that it computes the cutting forces. There are two general purposes that this work serves. The first of those purposes is: this work provides a base from which, the first iteration of the overall method of determining the probability of cutting tool failure, can be continued. In addition, by observing the results returned from the two models developed, conclusions can be made about the performance of the method.

The data generated from the two models described above verifies the programs that were written to implement the algorithm developed. The simple transformations used in model one, make the verification of some basic properties of this modeling process evident. The data from model one confirms several features which were built into the algorithm by it's specification. In addition, an unexpected but explainable feature is revealed in plots of the data. The unexpected feature that resulted from this algorithm, is that the direction of the cutting force changes when the chip volume is not relatively constant is size and shape. This change in the direction of the cutting force can be observed in the vector plots of both models, in the initial intersections of 
tool and workpiece. The second model is slightly more complex than the first. A visual inspection of the data verifies that the algorithm functioned in the manner in which it was intended to when applied to the second model. The results of the second model also show a change in the direction of the cutting forces in the first few time-steps.

The results of the algorithm developed here are written to a formatted data file as the components of the three dimensional forces acting on the nodes at discrete instances in time. These results will be used to approximate the amount of stress a cutting tool has undergone during a cutting process. In order to visualize the results, vectors of the forces have been plotted in two dimensions. The nodes of the finite element mesh are represented by dots, which make the outline of the tool somewhat discernible. In order to make the force vectors more visible, a scalar of ten (10) has been applied to all of the original data, in all views and time-steps, for both of the models discussed here. The views of the data are of the left side, back side and bottom side of the tool. The tip of the tool is called the front, the top of the tool is the top, and left and right sides are taken as if standing on the top of the tool looking in the direction of the tip. As shown in Figure 19, the top and right hand sides of the tool are visible, as well as the front end. 


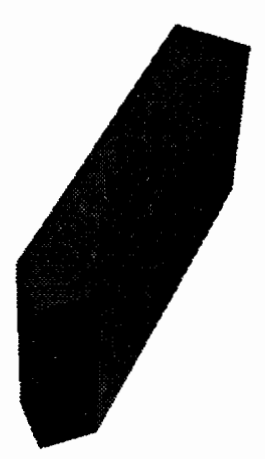

Figure 19 -Rendered image of the single lump version of the cutting tool.

The results are presented for both of the models in sets of five time-steps per page. Each row represents a discrete instance in time for which the forces acting on the tool were calculated. The time-steps are in consecutive order, starting with the first intersection of the cutting process modeled. In each row, three plots show the side, back, and bottom views of the tool, for the respective time-step. The data for the first model is presented, followed by a discussion of the rotational effect of the cutting force, and finally the results of the second model. The results of the second model are in the same format as the first. 


\subsection{Model One}

The first model differs from the second in several respects. One of the most notable differences concerns the transformations defined for the two models. Model one was a one degree of freedom motion. The tool swept through the workpiece, engaging and then disengaging the solid body, as shown in Figure 20.

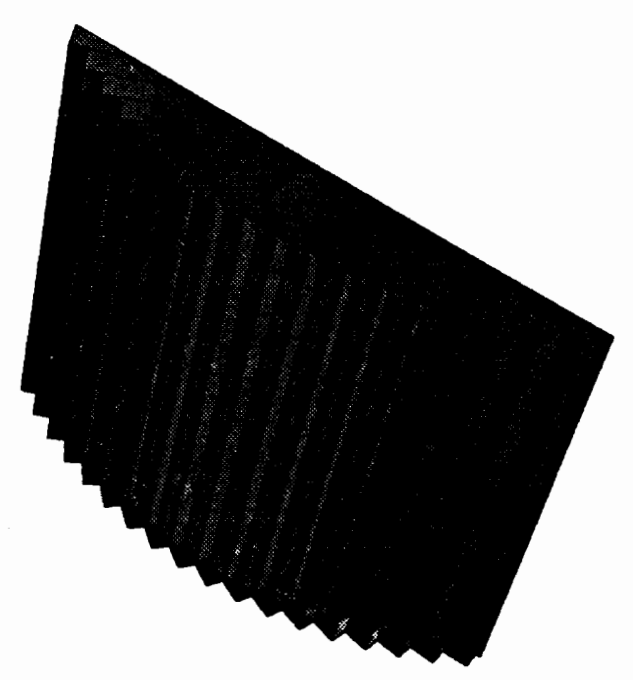

Figure 20 - Chips set generated from execution of Model One.

This motion caused the shape of the chips be vary at the ends of the process, and remain somewhat constant in the middle of the process. Model one was set up to complete the process with nineteen intersections of the tool and work piece.

Significant changes in the shape of the chips occurs in the first, and last, six or seven 
time-steps. This variation in shape causes the direction of the force vector to change between time-steps.

One of the most general verifications that the results correspond to the specifications of the model is that the direction of the force vectors varies consistently with the change in location of the centroid of the chips. In addition, the transformation of the tool was in one plane. This is evident in both the side and back views of the vector plots where the direction of the vectors is unchanged and in the plane of rotation. The simple transformation of the tool caused all of the changes in direction of the force vector to occur in the plane of the transformation. For this reason, we turn to the bottom view of the data for an analysis of the direction of the force vector.

The full engagement of the tool and work piece provides for a second simplification. Looking at the side and back views of the tool, we can see that forces are symmetric about a the middle, or fourth row of nodes in both of those views. Note that the magnitude of the forces acting on the nodes lying in the top and bottom plane of the cutting tool are roughly half of those that lie outside these planes. In the discussion of the methods by which the forces were calculated, the statement is made that the forces for nodes that have adjacent elements are summed. This observation is a verification of that statement. The calculation of the force acting on a node of the finite element mesh was based on the volume of the intersection of a solid representation of the element and a solid model of a chip. In these two models the same eight node brick 
elements were used to describe the tool mesh. The nodes this type of brick elements in a mesh can have from one to eight elements adjacent to it. Because of this, the forces which were calculated for the nodes lying in the top or bottom plane of the tool, were roughly half of the forces of the nodes lying outside the planes. This effect of the algorithm can also be seen in the second model. The increase in the complexity of the transformations in the second model do not make it as plainly evident, as in the first model.

In model one, the tool is rotated 4 degrees between time-steps. The bottom views of the force data, show an increase in the engagement of the tool and work piece as the model proceeds with each time-step. While the magnitude of the force on the nodes engaged stays relatively constant, the number of nodes intersecting the workpiece increases rapidly in the first four time-steps. The magnitudes of the forces stay relatively constant, for most of the nodes, because of the large transformation step size used in this case. A large initial transformations tends to saturate the nodal weights in one time-step. No detailed effort has been made to study the effect of changing the step size. The effect of elements being partially engaged can be seen in elements intersecting the parameter of the chip.

In addition to the number of nodes increasing between time-steps, the direction of the forces acting on the nodes during the initial time-steps changes. The change in direction of the calculated cutting force is due to the change in the shape of the chips 
during the initial period of the cutting process. The change in shape causes the center of mass of the chip to move, relative to the datum position, between time-steps. This effect is explained in the next section.

The layout of the first model in Figure 21, shows images of the cutting tool engaged with the work piece at the fifth time-step. This layout serves to communicate the orientation of the tool in the vector plots. The vector plots of the back and bottom views of the tool are rotated 90 degrees, in the clockwise direction, from the orientation of the tool in the layout.

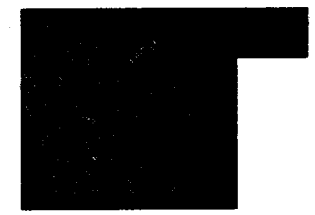

Side View

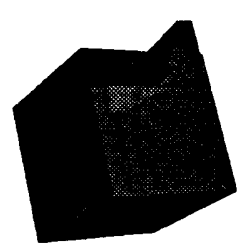

Model 1

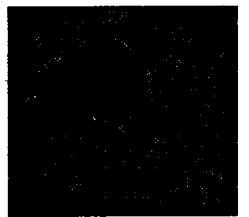

Back View

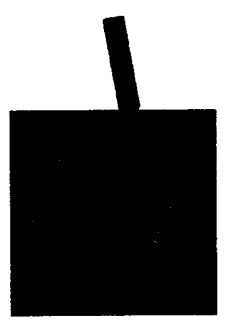

Bottom Vlew

Figure 21 - Layout of the views of the tool in the vector plots of the data generated by Model One. 

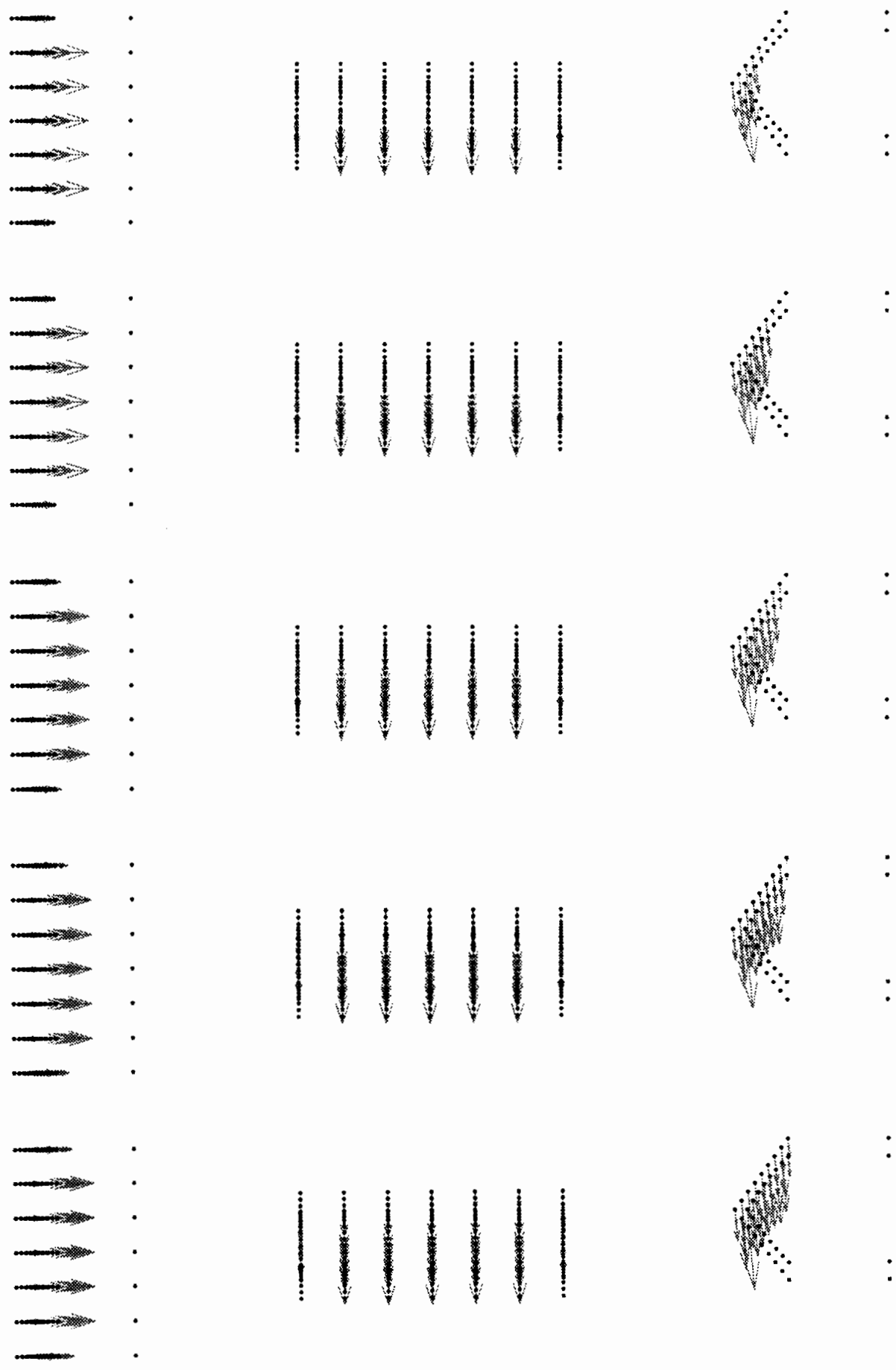

Figure 22 -Vector plots for the first five time-steps of Model One. 

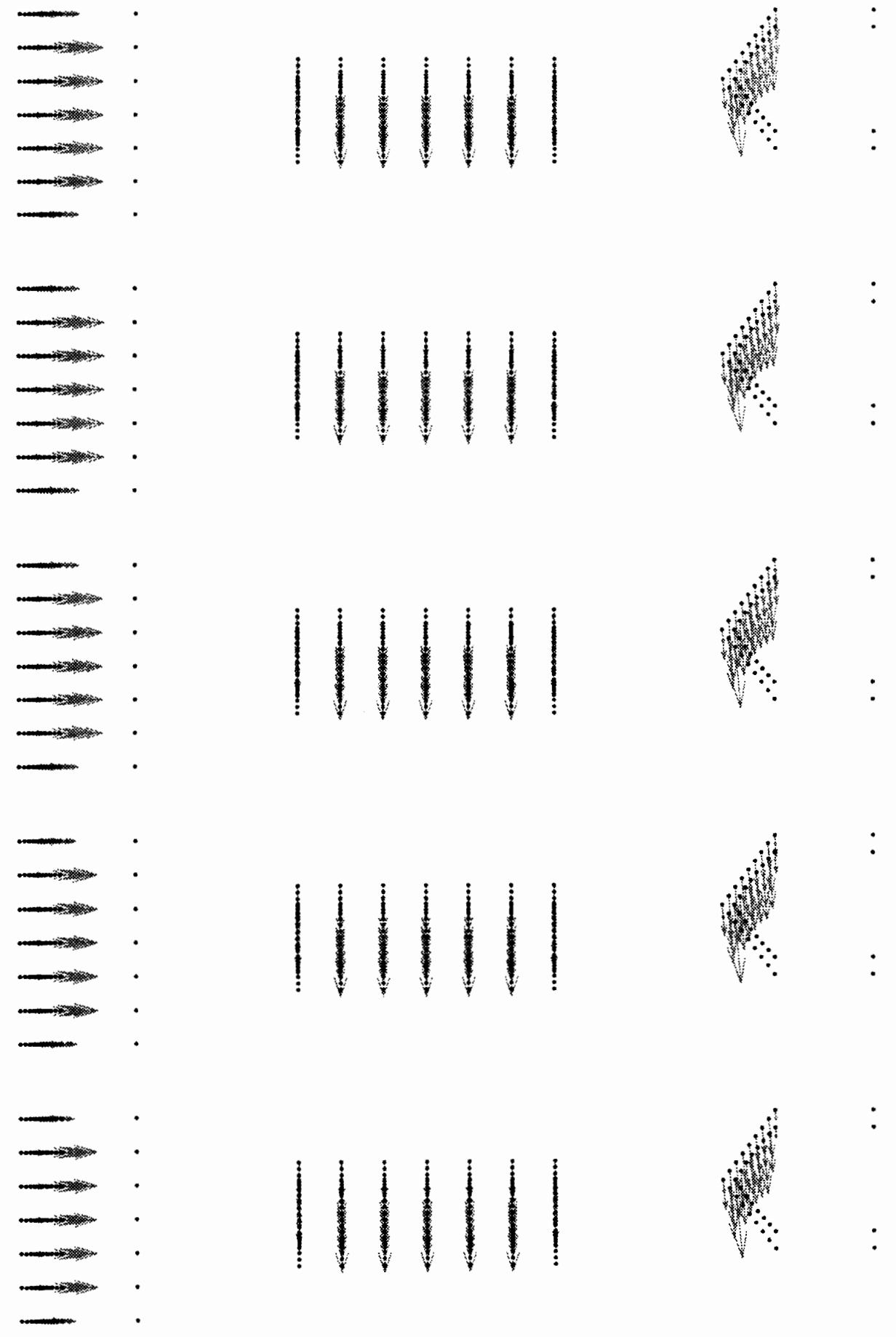

Figure 23 - Vector plots of calculated force data for Model One. Time-steps five through ten. 

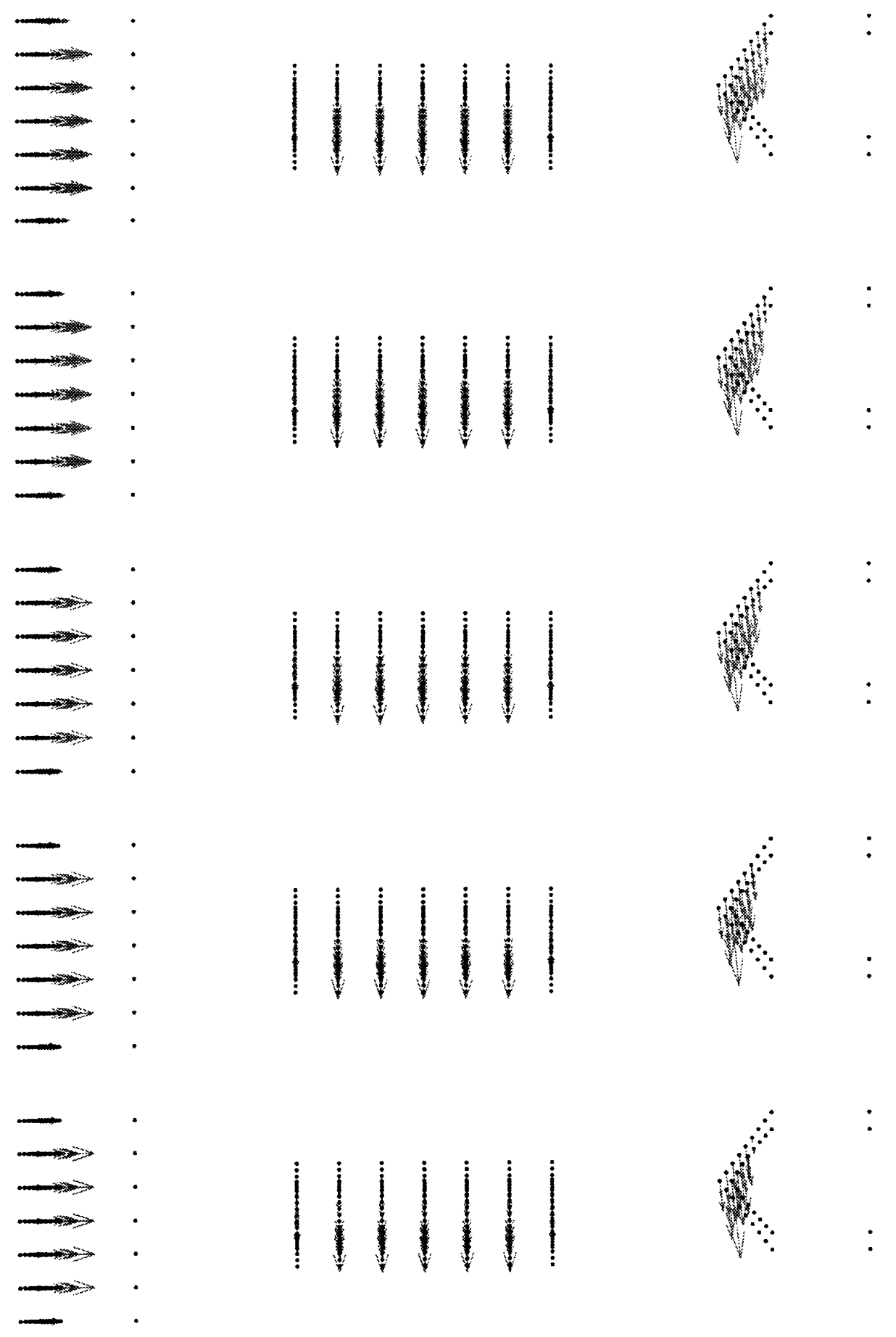

Figure 24 - Vector plots of calculated force data for Model One. Time-steps eleven through fifteen. 

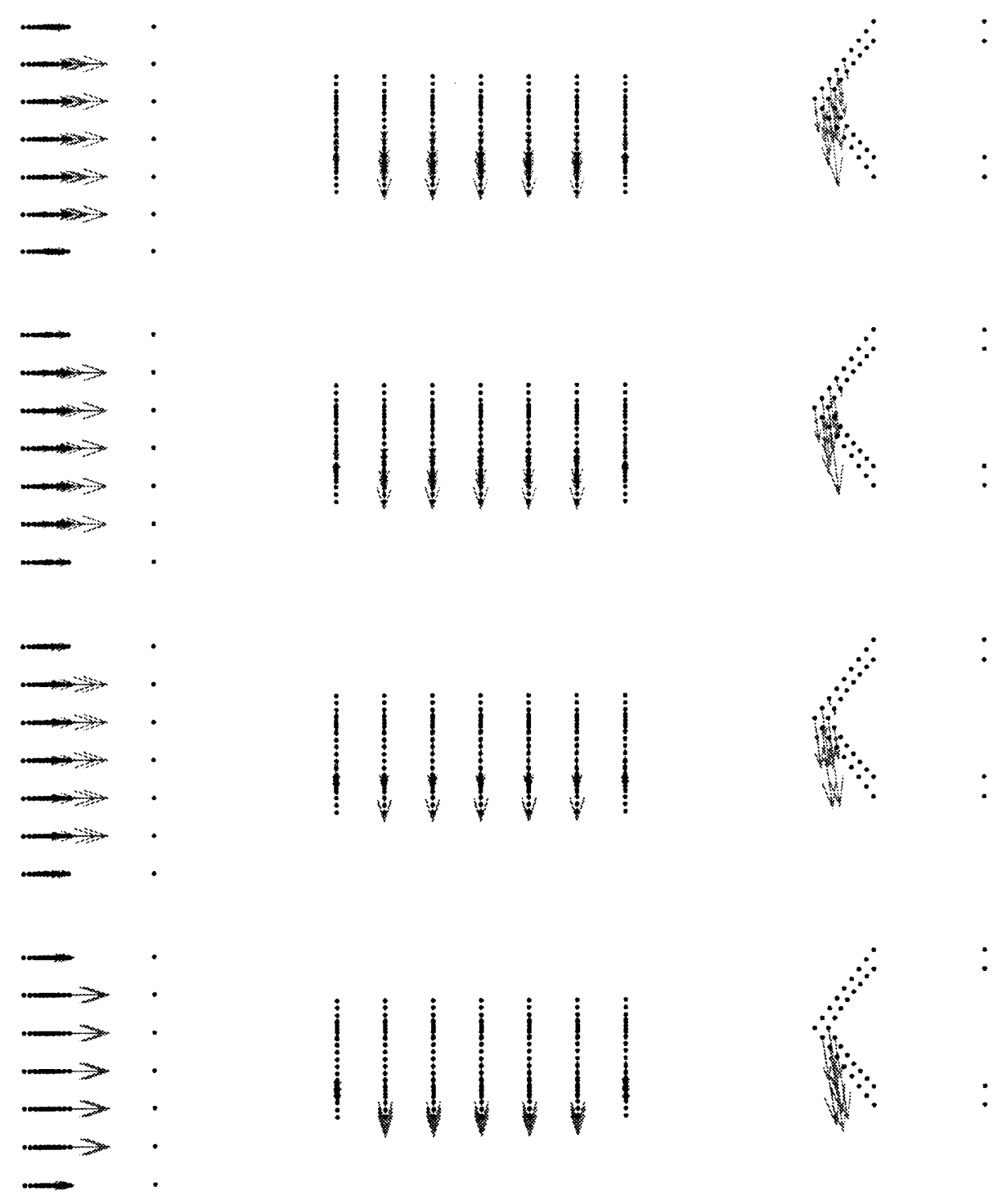

Figure 25 -Vector plots of calculated force data for Model One. Time-steps sixteen through nineteen. 
The last four time-steps of the first model, refer to Figure 25, show forces being applied to the trailing edge of the cutting tool. Although the application of forces on the trailing edge would not occur in a real world application of the type modeled, the forces are consistent with the definition of the model. The change in direction (ccw rotation) of the cutting forces as the tool exits the workpiece is also consistent with the algorithm and model specification.

\subsection{Change in Direction of Cutting Force}

The cutting forces that have been generated by both of these models, tend to change direction during different periods of the tool path. The change in direction of the cutting force is a side effect of the method by which the direction of the force was calculated. The models are discrete in nature and capture discrete instances of the direction vector changing. The frequency at which the process is sampled is high enough that aliasing of the change in direction does not occur in these two models. This algorithm solves for the forces based on the volume of the chip, and the direction based on the location of the centroid of that chip. As the cutting process proceeds, the centroids of the chip volumes shifts around in space. This shifting of the centroids is due to the change in shape and volume of the chips. When a change in the shape of the chips is relatively large between time-steps, the centroids of the chips may be located in different enough locations from each other, relative to the datum position, that the continuous shifting of the centroids is visible in the vector plots. In model one, both at 
the beginning of the cutting process and at the end, the direction of the force vectors change between time-steps. This change in direction appears as a rotation of the cutting force in the bottom views of the tool. The rotation of the direction vector in model two diminishes between time-steps until the tool is fully engaged with the work piece, at time-step eighteen. From the point at which the tool is fully engaged with the stock, the direction of the force vectors is relatively constant. The constant direction of the force vector occurs because the shape of the chips generated form the model are, for the most part, identical beyond time-step eighteen.

For a detailed description of the method by which the direction vector was computed please refer to The Direction of the Cutting Force discussion in the Force Calculation Algorithm section above. The rotation of the direction vector during the beginning of model one will be analyzed in some detail below, in order to explain the effect. This discussion refers to the first seven time-steps of model one as an example. The rotation of the force vectors can be most clearly seen by superimposing the results from time-steps one and seven, over the tool mesh. As shown in Figure 26, the darker arrows are the results of the force calculations for the first time-step, and the lighter arrows (of which there are many more, because the tool is more fully engaged) are the results of the seventh time-step. The rotation of the vectors is in the clockwise direction. 


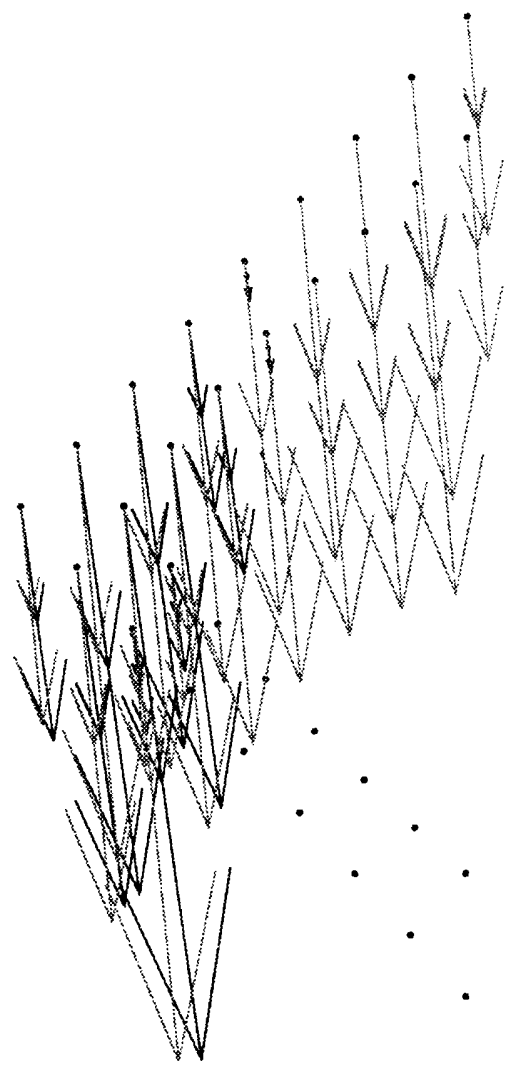

Figure 26 - Calculated force vectors for time-steps one and seven, of Model One, superimposed on the nodes of the tool mesh.

As mentioned above, the change in direction of the vectors is due to the change in the shape of the chips which occurs, in this case, at the start of the cutting process. As the shape and volume of the chips change between time-steps, the centroids, which are used to calculate the direction vector, change location relative to the datum position. Below is a two dimensional plot of the centroids of the chips which were generated in the first seven time-steps of model one. Shown in Figure 27 is a top view of the tool in the datum position. Each of the chips has been oriented on the datum position, so that the relative displacement of the centroids can be observed. As the tool 
engages the work piece, the centroids shift location from right to left. The centroid for the first time-step is at the location of the right-hand most ' 0 '. The order that the centroids occur is: $\mathrm{o}, \mathrm{x},+,{ }^{*}, \mathrm{o}, \mathrm{x},+$.

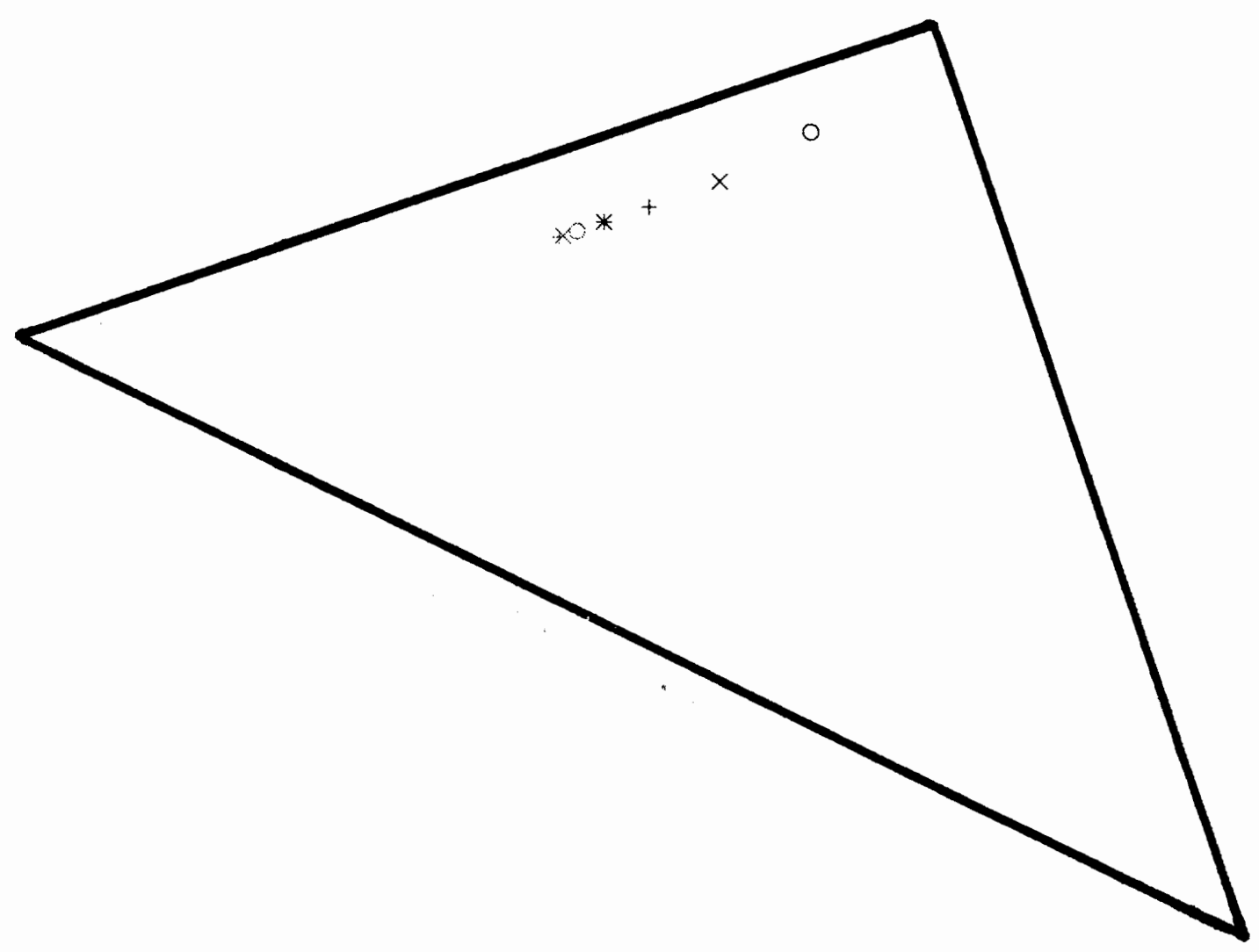

Figure 27 - Top view of the tip of the cutting tool with chip centroids, for timesteps one through seven, superimposed in the order $o, x,+, *, o, x,+$.

If the change in the location of the centroids were only along the radius of rotation of the tool, then the force vectors would be parallel for all time-steps. If this were true then there would be no relative rotation of the direction vectors. This is not 
the case. If we describe to positions of the centroids of the chips in polar coordinates, with the origin set at the center of rotation of the tool, then in addition to a radial change, the angular position of the centroids also changes. Figure 28 shows a top view of the tool with a radial line from the origin of a polar coordinate system to the tip the tool. 


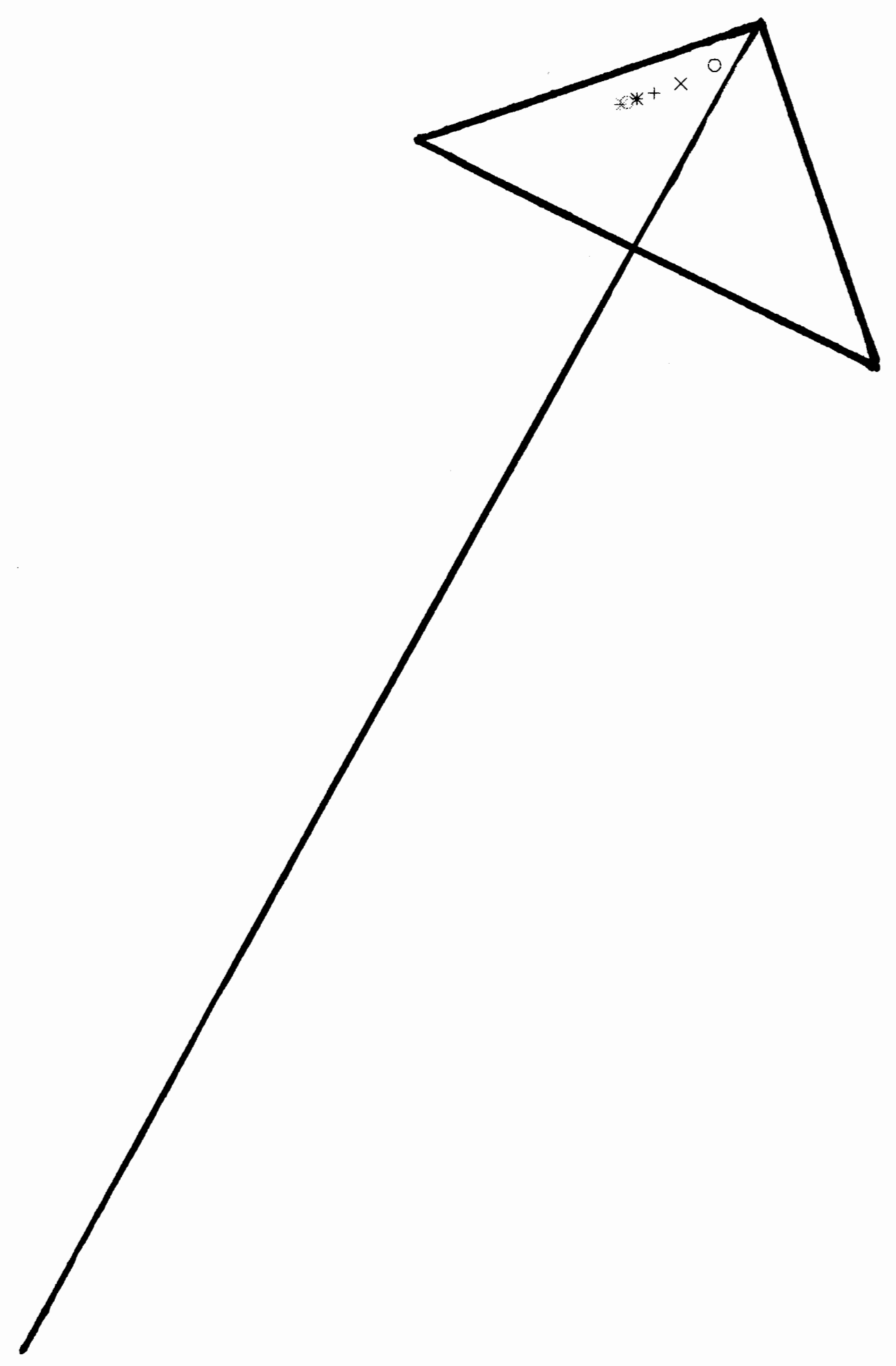

Figure 28 - First seven centroid locations for Model One, with a radial line shown from the origin of the polar coordinate system to the tip of the tool. 
Observe that the positions of the centroids experiences a rotation about the origin as well as a translation in the radial direction, between time-steps. The rotation of the direction vector is directly proportional to the rotation of the locations of the centroids shown above. The radial shift of centroids does not contribute to the change in direction of the force vector. The bottom views of the vector plots for model two (below) clearly display the clockwise rotation of the force vector as the tool engages the work piece. The same line of reasoning used to describe the rotation in model one could be followed with the second model.

\subsection{Model Two}

In the second model, the transformations that describe the relative motion of the cutting tool, take place about two degrees of freedom. The more complex tool path of this model, does not lend the results to some of the observations which were made in the first model. Among those observations, is the fact that there is no symmetrical distribution of the cutting forces. Instead, we see the vectors which have components in both dimensions of each of the three projections. Like the first model, the views of the tool are of the left side, back and bottom. A change in direction of the force vector is observed in two of the three projections. The side and bottom views of the data display a change in the direction of the cutting force as the tool engages the work piece. The change in direction is most evident in the bottom views of the force vectors. 
In the second model, we see a much more gradual penetration of the tool into the workpiece. Twenty time-steps worth of force data have been plotted for the second model. The tool is completely engaged with the work piece at time-step eighteen, and the data is relatively constant from that point in the path of the tool. The force vectors in all of the views of the results agree nicely with the specification of the model.

The layout of model two in Figure 29 shows the orientation of the tool in the vector plots. The plots of the back and bottom views of the tool are rotated clockwise 90 degrees from the orientation of the tool in the layout.

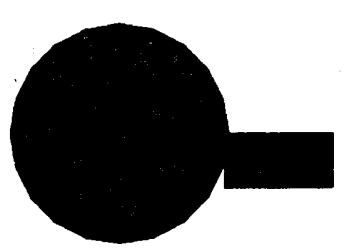

Side View

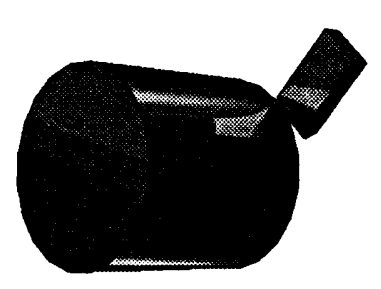

Model 2

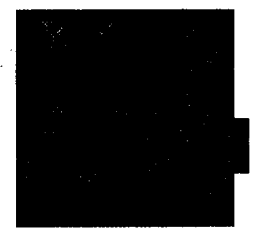

Back View

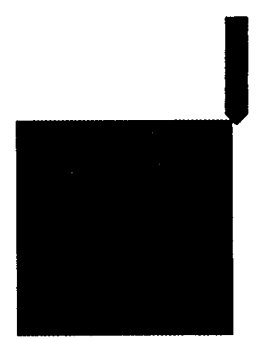

Bottom View

Figure 29 - Layout of the view of the cutting tool for Model Two. 

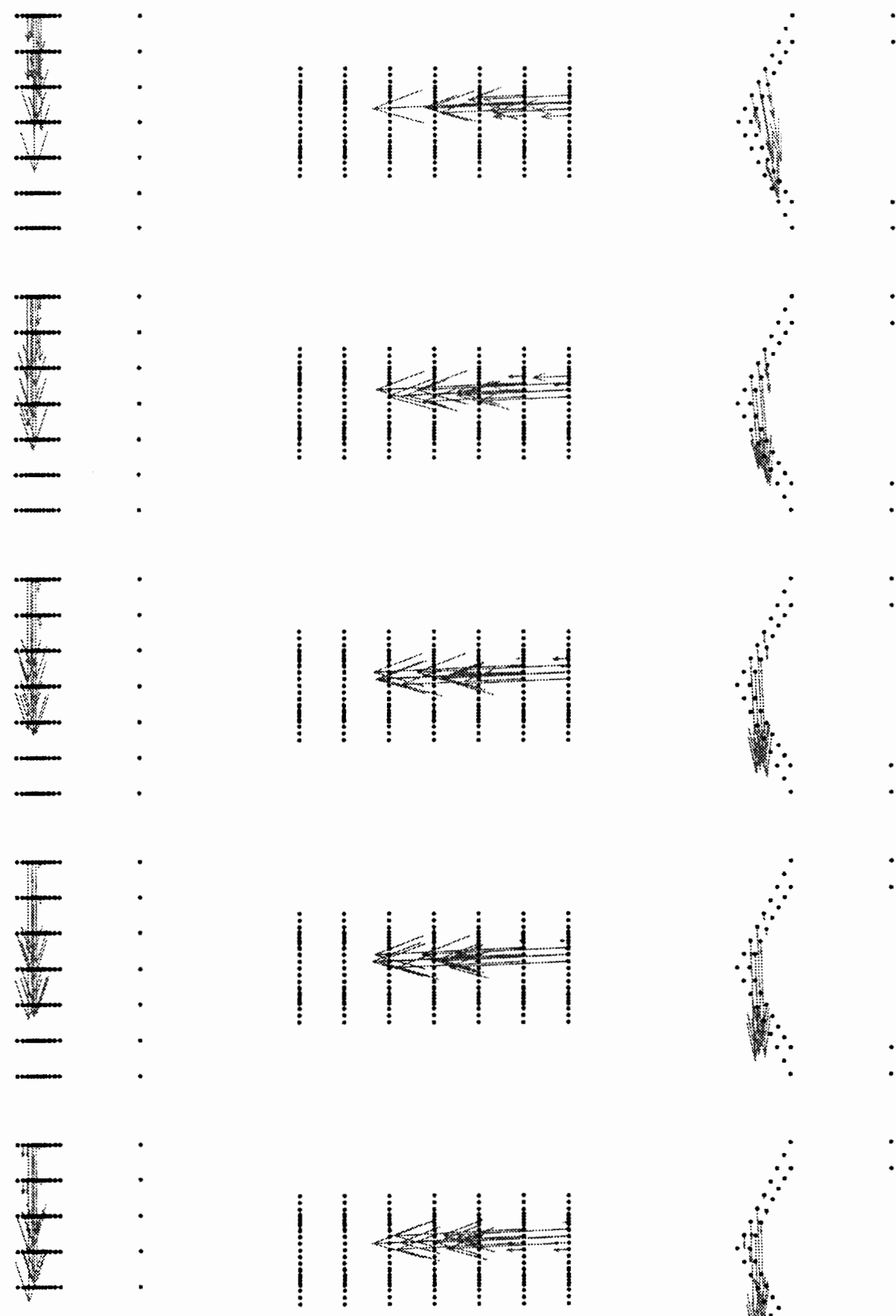

Figure 30 - Vector plots for the first five time-steps of Model Two. 

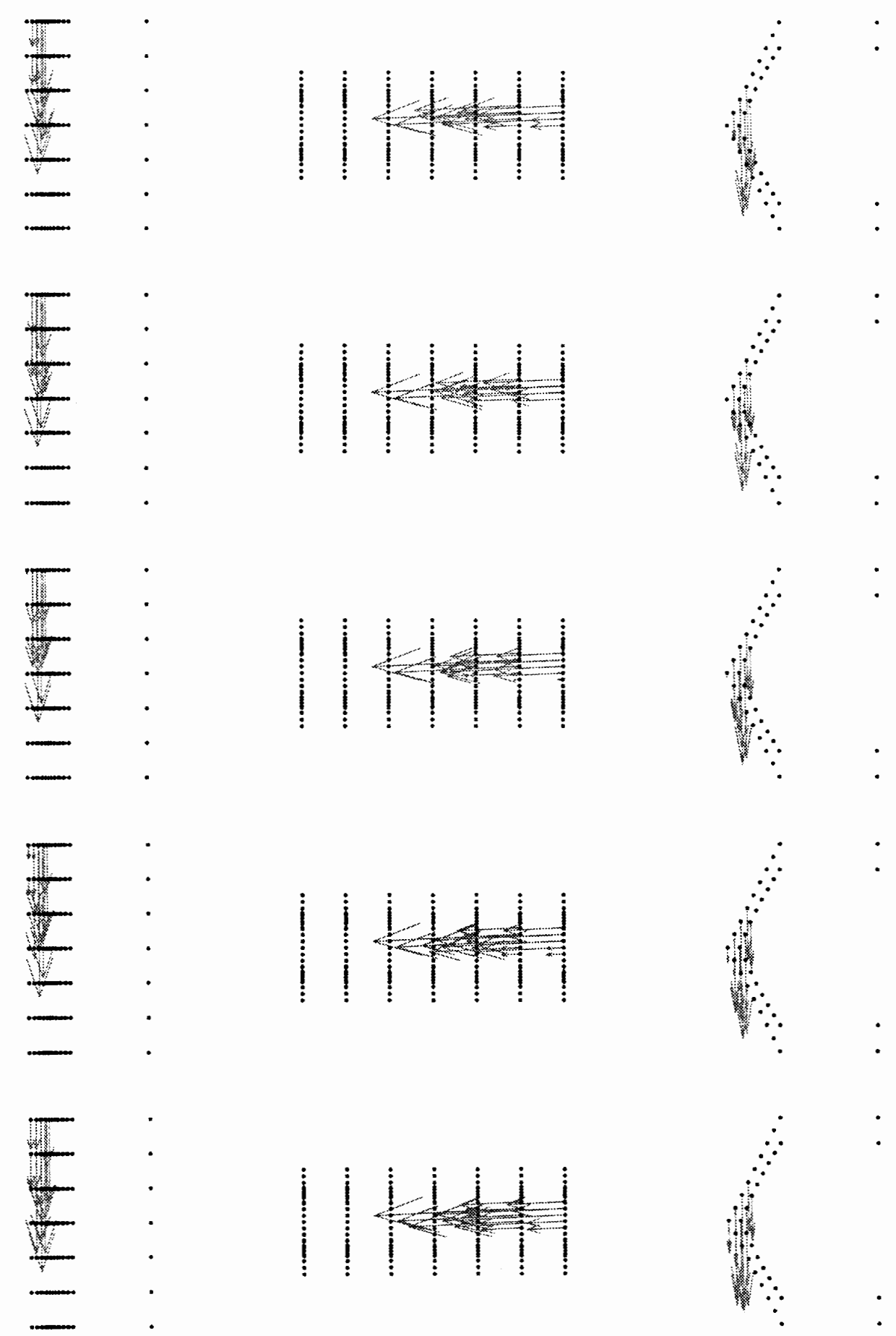

Figure 31 - Vector plots of calculated force data for Model Two. Time-steps six through ten. 

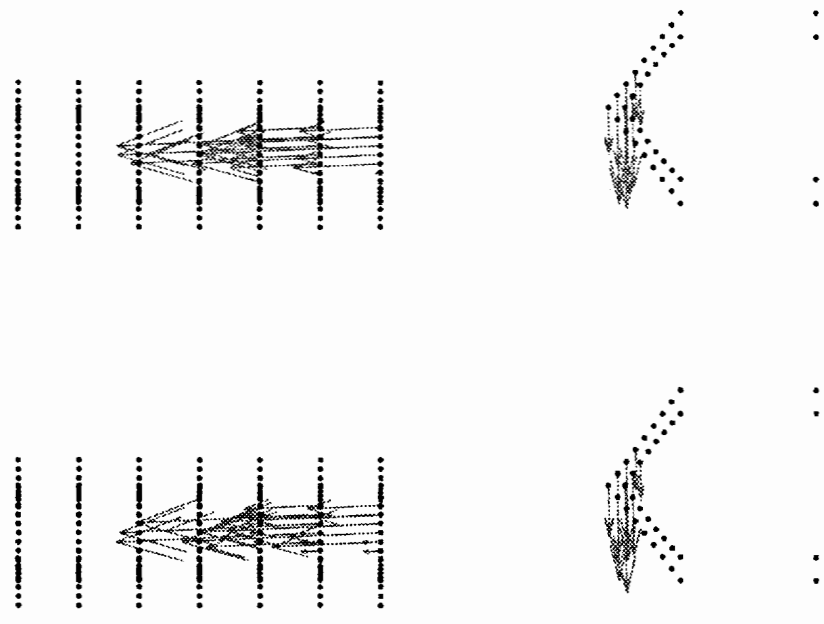

.
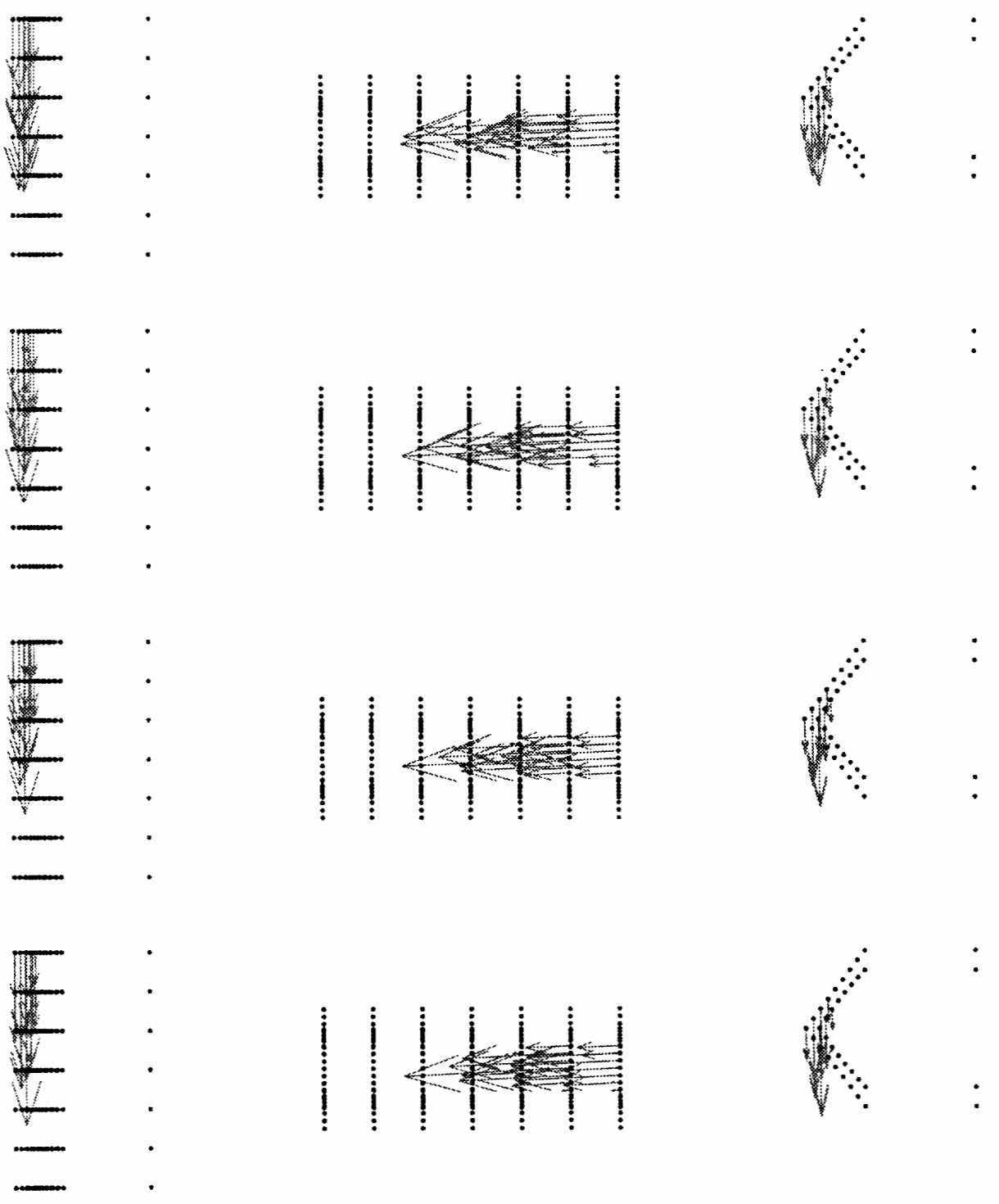

Figure 32 - Vector plots of calculated force data for Model Two. Time-steps eleven through fifteen. 

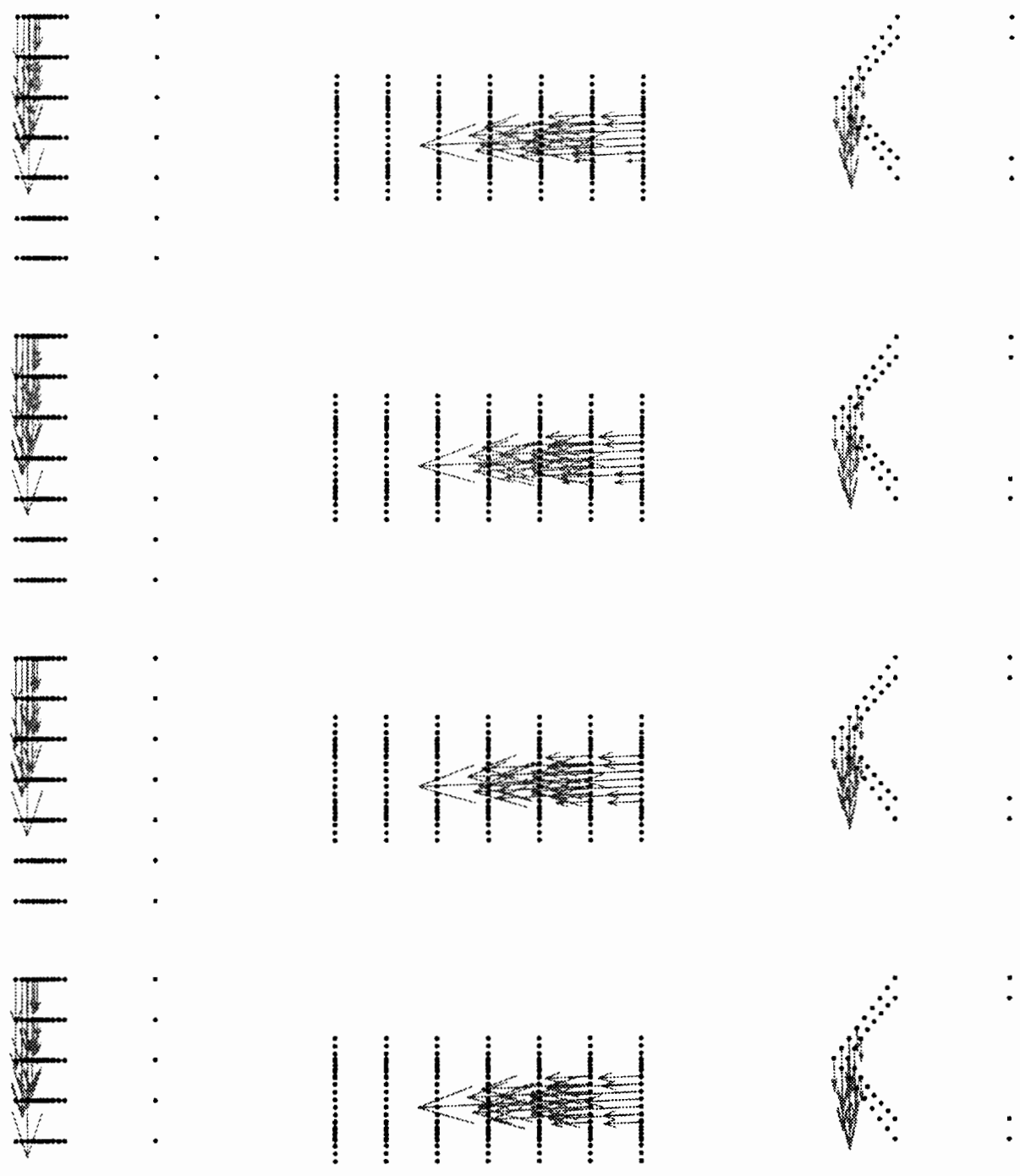

Figure 33 - Vector plots of calculated force data for Model Two. Time-steps sixteen through twenty. 


\subsection{Conclusion}

The algorithm outlined in this paper provide a base from which the overall algorithm to computationally determining the condition of cutting tools can be continued. This work begins an effort to develop an algorithm for determining the condition of cutting tools.

In addition to the work that needs to be done to complete the overall algorithm of tool condition monitoring, revisions need to be made to components of the algorithm described above. In spite of the fact that many simplifications were made, the results of this work are promising. With the continued use and increased power of computational hardware in the manufacturing process, a well developed method of computing the condition of cutting tools will be possible to implement on the shop floor. A method of this type may well be a standard feature on $\mathrm{CNC}$ machines of the future.

The simple approach of calculating the cutting forces served the purpose of moving the work forward. The method of approximating cutting forces is a first iteration that can be improved upon in the future. These results fulfill the original goal of this work by providing a method of determining cutting forces, that can be used in the broader goal of developing an algorithm for computationally determining the condition of cutting tools. There are several issues which are outside of the scope of this work and will need to be considered in the advancement of this process. Two of 
those issues are the effect that material properties and different step sizes, or frequency of intersection, have on the results.

Material properties were not accounted for in this work, therefore the forces calculated can be used in relative comparison but do not represent the absolute values of the forces. Future work would include the calculation of absolute force values that account for the effects of material properties.

The effect of changing the step size has not been thoroughly investigated in this work. If a similar line of reasoning is followed as was done in the analysis of the change in the direction of the force vector above, the conclusion can be made that a large step size would effect the calculation of the direction vector. A rough surface will result when a model is executed with a relatively large step size. If the step size is large enough, the surface created in a first pass could be rough enough that odd chip shapes could occur in the second pass. This in turn would cause the positions of the centroids of the set of chips made on the second pass to vary in an unrealistic manner. A varying of the centroids in this manner would certainly cause the direction of the force vectors to change. A possible solution to this problem would be to create a solid body by sweeping of the tool shape along a path that has been processed to create chips. This swept volume could then be used in a Boolean subtraction operation to cleanup the surface of the model so that the second set of chips would be consistent with the first. 
- A limitation of this implementation of a volumetric approach of calculating the forces, is that forces are distributed on nodes which are inside the surface of the tool. A solution to this problem would be to expand the current algorithm to solve for the forces based on the exterior surface area that is common to the chip and tool. Implementing an area approach to approximating the cutting forces would be similar to this implementation of the volumetric approach. An area approach would add complexity to the algorithm with the benefit of a possible improvement in the cutting force approximation. The nodal forces could be determined by dividing the area of the surface by the distance of the node to the centroid of the area, in a similar manner as was done in this work. This method could include a breakdown of the force calculations due friction as well as the shearing of material from the work piece. This, and other improvements, could make the forces calculated here a more accurate approximation of actual cutting forces.

The determination of the force direction may be improved by calculating an individual direction for each of the elements. This change could be made to the volumetric method described hear as well as to new methods. The direction of the force, in a surface area based method, could be based on a vector from the location of the centroid of the area at a time-step to the location of the centroid one time-step later. The desire to complete more of the tool condition monitoring algorithm, motivated the 
need to simplify the first iteration of the process, and develop this volumetric approach.

Like so many computer related problems, much time goes into the development of the software. After a problem such as this has been broken down and described in a program, a very powerful tool emerges that can be easily executed to produce or process large amounts of data. Perhaps one of the most dominant results of this work is the tool that has been created in the development of this algorithm. 


\section{References}

1 Rolt L.T.C., A Short History of Machine Tools, Massachusetts: M.I.T. Press, 1965, pp 17-91.

2 Boothroyd G., Knight W. A., Fundamentals of Machining and Machine Tools, New York: Marcel Dekker, 1989, p. 75

3 Tlusty J., Masood Z., Chipping and Breakage of Carbide Tools, New York: Journal of Engineering for Industry, 1978, vol. 100, pp. 403-412.

4 Sturges R. H., Monitoring Milling Processes Through AE and Tool/Part Geometry, New York: Journal of Engineering for Industry, 1992, Vol. 114, pp. 8-14.

5 Voelcker H.B., Hunt W A, The role of Solid Modeling in Machine-Process Modeling and NC Verification, SAE, 1981, 810195.

6 Chandrasekaran H., Thuvander A., Modeling of Tool Stresses in Peripheral Milling, Stockholm: Annals of the CIRP, 1988, Vol. 37/1/1988, pp 41-44.

7 Springer G., Friedman D.P., Scheme and The Art of Programming, Massachusetts: The MIT Press, 1994, p 129. 